Gene Ontology : biological process. Level: 3 neurological process

tissue remodeling

primary metabolic process

macromolecule metabolic process

response to abiotic stimulus

cell communication

behavior

cellular metabolic process

digestion

circulation

detection of stimulus

developmental growth

molting cycle

endocrine process

sleep

multicellular organismal homeostasis

organismal movement

death

catabolic process

regulation of a molecular function

coagulation

aging

response to stress

egulation of biological quality

localization of cell

thermoregulation

organ growth

cellular developmental process

autophagy

nomeostasis of number of cells

body growth

interspecies interaction between organisms

cell organization and biogenesis

defense response

cell proliferation
Percentage of genes with allele-specific expression

Percentage of genes without allele-specific expression 7.17

0.56

54.74

47.57

0.64

32.83

3.27

55.86

0.32

1.04

0.4

0.48

0.56

0.16

0.16

6.22

4.3

3.19

0.96

0.32

7.81

5.18

0.08

16.57

0.24

0.24

0.24

0.24

4.38

6.85
Unadjusted pvalue Adjusted pvalueFDR

\begin{tabular}{|c|c|}
\hline 2. & $1.73 \mathrm{E}-03$ \\
\hline 2.38 & $2.30 \mathrm{E}-03$ \\
\hline 48.16 & $1.65 \mathrm{E}-02$ \\
\hline 41.25 & 2.19E-02 \\
\hline 1.94 & $2.45 \mathrm{E}-02$ \\
\hline 38.66 & $2.56 \mathrm{E}-02$ \\
\hline 5.4 & $4.75 \mathrm{E}-02$ \\
\hline 50.76 & $6.35 \mathrm{E}-02$ \\
\hline 1.08 & $6.52 \mathrm{E}-02$ \\
\hline 2.16 & $9.48 \mathrm{E}-02$ \\
\hline 1.08 & $1.45 \mathrm{E}-01$ \\
\hline 0 & $2.00 \mathrm{E}-01$ \\
\hline 0 & $2.00 \mathrm{E}-01$ \\
\hline 0.22 & 2.69E-01 \\
\hline 0.22 & $2.69 \mathrm{E}-01$ \\
\hline 0.43 & $2.95 \mathrm{E}-01$ \\
\hline 0.43 & $2.95 \mathrm{E}-01$ \\
\hline 4.97 & 3.57E-01 \\
\hline 5.4 & 3.63E-01 \\
\hline 4.1 & $3.71 \mathrm{E}-01$ \\
\hline 0.43 & $3.76 \mathrm{E}-01$ \\
\hline 0.65 & 3.95E-01 \\
\hline 6.48 & $4.08 \mathrm{E}-01$ \\
\hline 1.3 & 4.20E-01 \\
\hline 4.1 & 4.49E-01 \\
\hline 0.22 & $4.66 \mathrm{E}-01$ \\
\hline 0.22 & $4.66 \mathrm{E}-01$ \\
\hline 15.12 & 5.07E-01 \\
\hline 0 & $5.68 \mathrm{E}-01$ \\
\hline 0 & $5.68 \mathrm{E}-01$ \\
\hline 0 & $5.68 \mathrm{E}-01$ \\
\hline 0 & $5.68 \mathrm{E}-01$ \\
\hline 17.06 & $5.72 \mathrm{E}-01$ \\
\hline 3.67 & $5.88 \mathrm{E}-01$ \\
\hline 7.56 & $5.97 \mathrm{E}-01$ \\
\hline
\end{tabular}


response to endogenous stimulus

cell activation

response to biotic stimulus

synapse organization and biogenesis

multicellular organismal development

establishment of localization

developmental maturation

cell homeostasis

chemical homeostasis

protein localization

cell adhesion

anatomical structure development

RNA localization

maintenance of localization

behavioral interaction between organisms

cell division

reproductive process

biosynthetic process

regulation of body fluids

cell recognition

nitrogen compound metabolic process

secondary metabolic process

respiratory gaseous exchange

cytokine production

pigmentation during development

cell cycle

membrane docking

sexual reproduction

chromosome segregation

extracellular matrix organization and biogenesis

circadian rhythm

excretion

\begin{tabular}{|c|c|}
\hline 4.54 & $6.20 \mathrm{E}-01$ \\
\hline 1.73 & $6.50 \mathrm{E}-01$ \\
\hline 3.67 & $6.54 \mathrm{E}-01$ \\
\hline 34.77 & 0.6912833 \\
\hline 1.94 & 0.7156244 \\
\hline 1.94 & 0.7157399 \\
\hline 2.59 & 0.7234653 \\
\hline 0.65 & 0.7345141 \\
\hline 21.6 & 0.7438192 \\
\hline 20.3 & 0.7890163 \\
\hline 0.86 & 0.7934371 \\
\hline 1.73 & 0.8442724 \\
\hline 2.16 & 0.8518755 \\
\hline 6.05 & 0.9107316 \\
\hline 7.78 & 0.918937 \\
\hline 21.6 & 0.9471706 \\
\hline 0.22 & 1 \\
\hline 0 & 1 \\
\hline 0.22 & 1 \\
\hline 1.51 & 1 \\
\hline 1.51 & 1 \\
\hline 8.86 & 1 \\
\hline 0.86 & 1 \\
\hline 0.43 & 1 \\
\hline 2.59 & 1 \\
\hline 0.22 & 1 \\
\hline 0.22 & 1 \\
\hline 1.08 & 1 \\
\hline 0.22 & 1 \\
\hline 6.91 & 1 \\
\hline 0.22 & 1 \\
\hline 1.94 & 1 \\
\hline 0.43 & 1 \\
\hline 0.43 & 1 \\
\hline 0.22 & \\
\hline 0.22 & \\
\hline
\end{tabular}


Gene Ontology : biological process. Level: 4 protein metabolic process

\section{sensory perception}

cellular macromolecule metabolic process

bone remodeling

biopolymer metabolic process

adult behavior

phosphorus metabolic process

detection of external stimulus

positive regulation of biological process

detection of abiotic stimulus

glucose homeostasis

rhythmic behavior

regulation of binding

response to radiation

regulation of locomotion

learning and/or memory

response to organic substance

\section{cell aging}

signal transduction

regulation of growth

response to wounding

regulation of blood vessel size

smooth muscle contraction

drug metabolic process

hair cycle

molting cycle process

regulation of multicellular organismal process

embryonic development

cell-cell signaling

cellular catabolic process

response to gravity

homoiothermy

response to mechanical stimulus

chemosensory behavior

striated muscle contraction
Percentage of genes with allele-specific expression Percentage of genes without allele-specific expression

Unadjusted pvalue Adjusted pvalueFDR

\begin{tabular}{|c|c|c|}
\hline 27.54 & 20.88 & 0.005665717 \\
\hline 3.85 & 6.81 & 0.01311375 \\
\hline 26.15 & 20.44 & 0.01545811 \\
\hline 0.57 & 1.98 & 0.01897887 \\
\hline 38.36 & 32.53 & 0.03037133 \\
\hline 0.25 & 1.1 & 0.03826845 \\
\hline 9.43 & 6.15 & 0.03852618 \\
\hline 0.16 & 0.88 & 0.04988037 \\
\hline 11.31 & 8.13 & 0.05967504 \\
\hline 0.08 & 0.66 & 0.06361406 \\
\hline 0.08 & 0.66 & 0.06361406 \\
\hline 0 & 0.44 & 0.07367107 \\
\hline 0 & 0.44 & 0.07367107 \\
\hline 0.41 & 1.32 & 0.07998575 \\
\hline 0.49 & 1.32 & 0.09926941 \\
\hline 0.82 & 1.76 & 0.111236 \\
\hline 0.16 & 0.66 & 0.1273231 \\
\hline 0.16 & 0.66 & 0.1273231 \\
\hline 30.25 & 34.07 & 0.1388143 \\
\hline 1.64 & 0.66 & 0.1583784 \\
\hline 3.93 & 2.42 & 0.1787818 \\
\hline 0.08 & 0.44 & 0.1811172 \\
\hline 0.08 & 0.44 & 0.1811172 \\
\hline 0.08 & 0.44 & 0.1811172 \\
\hline 0.57 & 0 & 0.2000642 \\
\hline 0.57 & 0 & 0.2000642 \\
\hline 2.87 & 4.18 & 0.2123753 \\
\hline 3.69 & 2.42 & 0.2238763 \\
\hline 7.05 & 8.79 & 0.2517451 \\
\hline 3.77 & 5.05 & 0.2683857 \\
\hline 0 & 0.22 & 0.2716418 \\
\hline 0 & 0.22 & 0.2716418 \\
\hline 0 & 0.22 & 0.2716418 \\
\hline 0 & 0.22 & 0.2716418 \\
\hline 0.16 & 0.44 & 0.2986204 \\
\hline
\end{tabular}


interleukin-6 production

response to drug

alkene metabolic process

locomotory behavior

cell motility

organelle organization and biogenesis

macromolecule catabolic process

response to other organism

nucleobase, nucleoside, nucleotide and nucleic acid met

response to protein stimulus

cell differentiation

alcohol metabolic process

interferon-gamma production

detection of chemical stimulus

response to temperature stimulus

response to pain

musculoskeletal movemen

sulfur metabolic process

anatomical structure morphogenesis

oxygen and reactive oxygen species metabolic process

reproductive developmental process

cofactor metabolic process

immune response

appendage development

pattern specification process

negative regulation of biological process

regulation of cellular process

regulation of coagulation

response to hormone stimulus

\begin{tabular}{|c|c|}
\hline 0.44 & 0.2986204 \\
\hline 0.44 & 0.2986204 \\
\hline 0.44 & 0.2986204 \\
\hline 0 & 0.3318527 \\
\hline 0 & 0.3318527 \\
\hline 0.66 & 0.3535703 \\
\hline 0.66 & 0.3535703 \\
\hline 2.86 & 0.367473 \\
\hline 4.18 & 0.3797009 \\
\hline 7.91 & 0.3878559 \\
\hline 3.52 & 0.4150318 \\
\hline 2.42 & 0.4213928 \\
\hline 23.3 & 0.4448913 \\
\hline 0.22 & 0.4584797 \\
\hline 15.38 & 0.4604103 \\
\hline 1.76 & 0.4659934 \\
\hline 0.22 & 0.4696125 \\
\hline 0.22 & 0.4696125 \\
\hline 0.22 & 0.4696125 \\
\hline 0.22 & 0.4696125 \\
\hline 0.22 & 0.4696125 \\
\hline 0.88 & 0.4741691 \\
\hline 10.11 & 0.4843789 \\
\hline 0.88 & 0.5027782 \\
\hline 0.88 & 0.5027782 \\
\hline 1.1 & 0.5031182 \\
\hline 5.27 & 0.5180336 \\
\hline 0.44 & 0.5305785 \\
\hline 1.32 & 0.5307622 \\
\hline 10.77 & 0.5491735 \\
\hline 30.33 & 0.5544919 \\
\hline 0 & 0.5672571 \\
\hline 0 & 0.5672571 \\
\hline 0 & 0.5672571 \\
\hline 0 & 0.5672571 \\
\hline 0 & 0.5672571 \\
\hline
\end{tabular}


interleukin-2 production

fear response

aromatic compound metabolic process

regulation of catalytic activity

response to xenobiotic stimulus

organic acid metabolic process

gametogenesis

response to DNA damage stimulus

leukocyte activation

heterocycle metabolic process

vitamin metabolic process

cell cycle process

hormone metabolic process

one-carbon compound metabolic process

regulation of developmental process

hemostasis

lipid metabolic process

cellular localization

tube development

ion homeostasis

transport

amino acid and derivative metabolic process

membrane organization and biogenesis

cell-cell adhesion

carbohydrate metabolic process

macromolecule complex assembly

system development

mitotic cell cycle

response to toxin

response to inorganic substance

maintenance of protein localization

cellular biosynthetic process

\begin{tabular}{|c|c|}
\hline 20 & 0.5765539 \\
\hline 0 & 0.5796803 \\
\hline 0 & 0.5796803 \\
\hline 0.44 & 0.6173481 \\
\hline 0.44 & 0.6173481 \\
\hline 0.44 & 0.6173481 \\
\hline 1.1 & 0.6450092 \\
\hline 3.74 & 0.6506001 \\
\hline 0.44 & 0.6656493 \\
\hline 4.18 & 0.6677004 \\
\hline 1.54 & 0.6855553 \\
\hline 1.76 & 0.7016256 \\
\hline 1.98 & 0.7149152 \\
\hline 0.44 & 0.7371745 \\
\hline 0.44 & 0.7371745 \\
\hline 6.81 & 0.7373835 \\
\hline 0.88 & 0.744756 \\
\hline 0.88 & 0.744756 \\
\hline 2.86 & 0.7547742 \\
\hline 0.66 & 0.771524 \\
\hline 5.49 & 0.8078098 \\
\hline 5.71 & 0.8099349 \\
\hline 1.1 & 0.8106824 \\
\hline 1.54 & 0.8360171 \\
\hline 20.22 & 0.8387658 \\
\hline 1.98 & 0.8518027 \\
\hline 2.64 & 0.8638721 \\
\hline 3.08 & 0.8726646 \\
\hline 3.3 & 0.8817467 \\
\hline 3.96 & 0.8862178 \\
\hline 18.68 & 0.8886906 \\
\hline 1.76 & 1 \\
\hline 0 & 1 \\
\hline 0 & 1 \\
\hline 0 & 1 \\
\hline 8.35 & 1 \\
\hline
\end{tabular}


generation of precursor metabolites and energy

cellular structure disassembly

fertilization

cell-cell recognition

interleukin-1 production

viral reproductive process

reproductive process in a multicellular organism

chemokine production

pigment metabolic process

nitric oxide metabolic process

smooth muscle cell proliferation

response to tumor cell

cytoplasm organization and biogenesis

amine metabolic process

urea cycle intermediate metabolic process

secretion

establishment of protein localization

collagen fibril organization

cholesterol homeostasis

response to osmotic stress

blood pressure regulation

endothelial cell proliferation

keratinocyte proliferation

response to hypoxia

reproductive behavio

interleukin-12 production

cellular defense response

cell redox homeostasis

neuromuscular process

regulation of cytokine production

peptide metabolic process

sequestering of metal ion

\begin{tabular}{|c|c|c|}
\hline 0.44 & 1 & 1 \\
\hline 0 & 1 & 1 \\
\hline 0 & 1 & 1 \\
\hline 0 & 1 & 1 \\
\hline 3.52 & 1 & 1 \\
\hline 0.22 & 1 & 1 \\
\hline 0.22 & 1 & 1 \\
\hline 0 & 1 & 1 \\
\hline 0 & 1 & 1 \\
\hline 0.22 & 1 & 1 \\
\hline 0.44 & 1 & 1 \\
\hline 0 & 1 & 1 \\
\hline 0 & 1 & 1 \\
\hline 0.22 & 1 & 1 \\
\hline 0 & 1 & 1 \\
\hline 0 & 1 & 1 \\
\hline 0.22 & 1 & 1 \\
\hline 2.64 & 1 & 1 \\
\hline 0 & 1 & 1 \\
\hline 2.86 & 1 & 1 \\
\hline 5.93 & 1 & 1 \\
\hline 0.22 & 1 & 1 \\
\hline 0 & 1 & 1 \\
\hline 0 & 1 & 1 \\
\hline 0.22 & 1 & 1 \\
\hline 0.22 & 1 & 1 \\
\hline 0 & 1 & 1 \\
\hline 0 & 1 & 1 \\
\hline 0.22 & 1 & 1 \\
\hline 0.22 & 1 & 1 \\
\hline 0.22 & 1 & 1 \\
\hline 0 & 1 & 1 \\
\hline 0.22 & 1 & 1 \\
\hline 0 & 1 & 1 \\
\hline 0.22 & 1 & 1 \\
\hline 0 & 1 & 1 \\
\hline
\end{tabular}


detection of biotic stimulus

ribonucleoprotein complex biogenesis and assembly

interleukin-4 production

osmoregulation

detection of endogenous stimulus

neuron recognition

granulocyte macrophage colony-stimulating factor produ

cell-substrate adhesion

regulation of gene expression, epigenetic

asymmetric cell division

tumor necrosis factor-beta production

metabolic compound salvage

entrainment of circadian clock

interleukin-3 production

grooming behavior

external encapsulating structure organization and bioge

behavioral defense response

$\begin{array}{rll}0 & 1 & 1 \\ 0 & 1 & 1 \\ 0.44 & 1 & 1 \\ 0.22 & 1 & 1 \\ 0.22 & 1 & 1 \\ 0.88 & 1 & 1 \\ 0 & 1 & 1 \\ 0 & 1 & 1 \\ 0.22 & 1 & 1 \\ 0 & 1 & 1 \\ 0.22 & 1 & 1 \\ 0 & 1 & 1 \\ 0.44 & 1 & 1 \\ 0.22 & 1 & 1 \\ 0 & 1 & 1 \\ 0 & 1 & 1 \\ 0 & 1 & 1 \\ 0 & 1 & 1 \\ 0 & 1 & 1 \\ 0 & 1 & 1 \\ 0 & 1 & 1 \\ 0.22 & 1 & 1\end{array}$


Gene Ontology : biological process. Level: 5 biopolymer modification

sensory perception of chemical stimulus

cell surface receptor linked signal transduction

cellular protein metabolic process

regulation of bone remodeling

phosphate metabolic process

sensory perception of light stimulus

response to amphetamine

vasoconstriction

positive regulation of cellular process

polysaccharide metabolic process

transmission of nerve impulse

regulation of cell adhesion

nitrogen compound biosynthetic process

female gamete generation

sulfur compound biosynthetic process

regulation of transferase activity

urogenital system development

response to light stimulus

negative regulation of multicellular organismal process

detection of stimulus during sensory perception

regulation of cell motility

esponse to bacterium

cell fate commitment

embryonic development (sensu Metazoa)

biopolymer catabolic process

hair cycle process

water-soluble vitamin metabolic process

nucleoside metabolic process

nuclear organization and biogenesis

intracellular signaling cascade

flammatory response

cellular macromolecule catabolic process

positive regulation of metabolic process

ion transport
Percentage of genes with allele-specific expression

Percentage of genes without allele-specific expression

Unadjusted pvalue Adjusted pvalueFDR

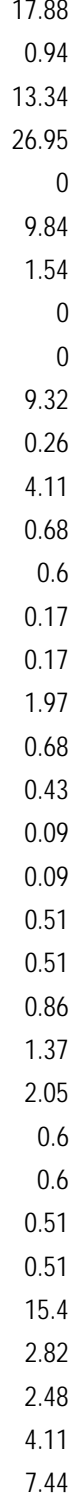

0.000946105

0.004519434

0.008701017

0.01376415

0.01944723

0.03816538

0.06615464

0.07243981

0.07243981

0.08640517

0.08936988

0.1083607

0.1176141

0.1246316

0.1246602

0.1246602

0.1359861

0.1379155

0.1450318

0.178425

0.178425

0.1786355

0.1786355

0.1802032

0.1812811

0.1938639

0.1999694

0.1999694

0.2000239

0.2000239

0.2030225

0.2079993

0.2314653

0.2386537

0.2512664
1

(1)

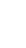

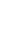


fast regulation of arterial pressure

sequestering of lipid

antibiotic metabolic process

retinal homeostasis

urothelial cell proliferation

detection of gravity

microtubule organizing center organization and biogene؛

response to ethanol

dosage compensation

organic acid catabolic process

regulation of posture

exocrine system development

eating behavior

parturition

circadian behavior

regulation of DNA binding

nervous system development

cellular lipid metabolic process

response to fungus

fat-soluble vitamin metabolic process

mesenchymal cell differentiation

photoreceptor cell differentiation

hormone biosynthetic process

calcium-dependent cell-cell adhesion

egulation of catabolic process

alcohol catabolic process

cytoskeleton organization and biogenesis

regulation of heart contraction

\begin{tabular}{|c|c|}
\hline 0.46 & 0.2619532 \\
\hline 0.23 & 0.269375 \\
\hline 0.23 & 0.269375 \\
\hline 0.23 & 0.269375 \\
\hline 0.23 & 0.269375 \\
\hline 0.23 & 0.269375 \\
\hline 0.23 & 0.269375 \\
\hline 0.23 & 0.269375 \\
\hline 0.23 & 0.269375 \\
\hline 0.23 & 0.269375 \\
\hline 0.23 & 0.269375 \\
\hline 0.23 & 0.269375 \\
\hline 0.23 & 0.269375 \\
\hline 0.23 & 0.269375 \\
\hline 0.23 & 0.269375 \\
\hline 0.23 & 0.269375 \\
\hline 0.23 & 0.269375 \\
\hline 0.23 & 0.269375 \\
\hline 0.23 & 0.269375 \\
\hline 0.23 & 0.269375 \\
\hline 0.23 & 0.269375 \\
\hline 0.23 & 0.269375 \\
\hline 0.23 & 0.269375 \\
\hline 8.12 & 0.290489 \\
\hline 5.8 & 0.291837 \\
\hline 0.46 & 0.2946967 \\
\hline 0.46 & 0.2946967 \\
\hline 0.46 & 0.2946967 \\
\hline 0.46 & 0.2946967 \\
\hline 0.46 & 0.2946967 \\
\hline 0.46 & 0.2946967 \\
\hline 0.46 & 0.2946967 \\
\hline 0.23 & 0.3044141 \\
\hline 4.41 & 0.3204322 \\
\hline 1.16 & 0.3239519 \\
\hline 0 & 0.3323761 \\
\hline
\end{tabular}

regulation of cyclase activity 
DNA damage response, signal transduction

mitochondrion organization and biogenesis

regulation of signal transduction

response to oxidative stress

lipid catabolic process

regulation of immune system process

reproductive structure development

cell morphogenesis

membrane invagination

organ development

regionalization

sensory perception of mechanical stimulus

cell development

heterophilic cell adhesion

amine transport

regulation of secretion

response to unfolded protein

endosome organization and biogenesis

Golgi organization and biogenesis

vesicle organization and biogenesis

superoxide metabolic process

aromatic compound biosynthetic process

bone resorption

digestive process

regulation of balance

detection of calcium ion

cell activation during immune response

negative regulation of locomotion

calcium-independent cell-cell adhesion

response to cold

regulation of oxidoreductase activity

glycerol ether biosynthetic process

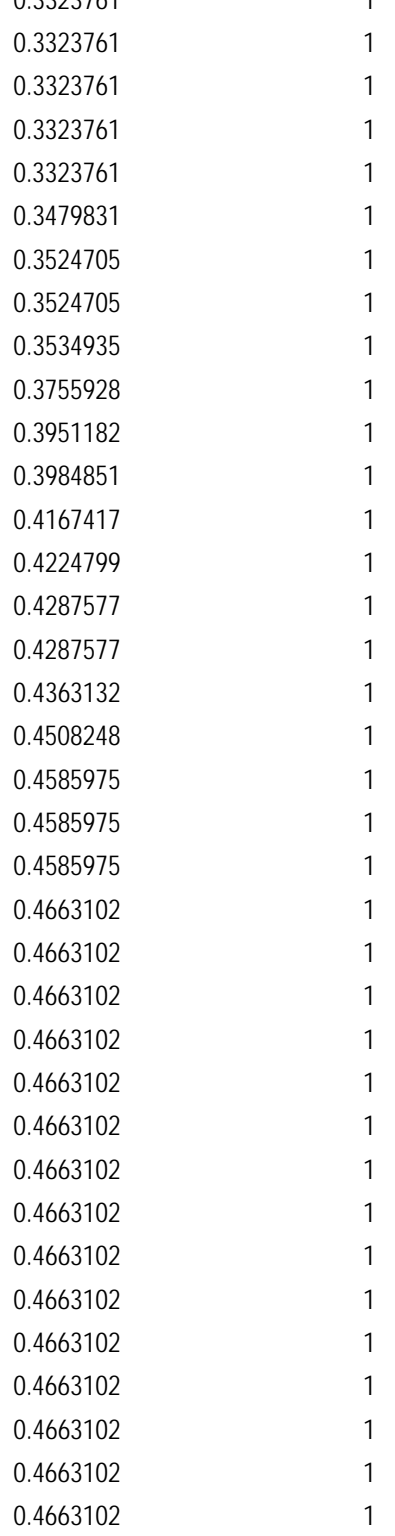


pteridine and derivative metabolic process

sex determination

terpene metabolic process

sensory perception of temperature stimulus

localization within membrane

coenzyme metabolic process

sex differentiation

protein-DNA complex assembly

morphogenesis of a branching structure

learning

regulation of anatomical structure morphogenesis

lipid transport

appendage morphogenesis

embryonic pattern specification

nucleotide metabolic process

lymphocyte activation

negative regulation of developmental process

mmune effector process

regulation of transport

regulation of cell activation

regulation of cellular metabolic process

regulation of body size

negative regulation of coagulation

nucleobase metabolic process

axis specification

alcohol biosynthetic process

regulation of defense response

gene silencing

interaction with host

lactation

negative regulation of metabolic process

cell migration

RNA metabolic process

0.23
0.23
0.23
0.23
0.23
0.23
0.23
0.23
0.93
0.93
0.93
0.93
0.93
0.93
0.46
0.46
0.46
1.39
1.62
1.16
1.16
1.16
1.16
20.19
0
0
0
0
0
0
0
0
3.48
18.33

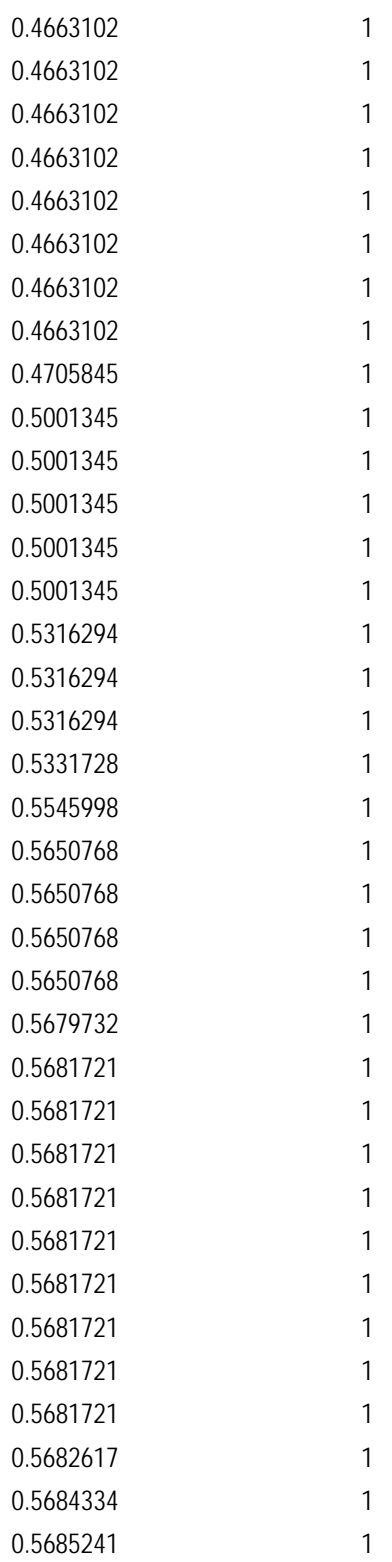


immune response-regulating signal transduction

secretory pathway

negative regulation of cellular process

chromosome organization and biogenesis

peptide transport

regulation of muscle contraction

vacuole organization and biogenesis

wound healing

regulation of cell proliferation

regulation of cell cycle

plasma membrane organization and biogenesis

xenobiotic metabolic process

nitrogen compound catabolic process

myeloid leukocyte activation

carboxylic acid metabolic process

embryonic morphogenesis

cell ion homeostasis

at cell differentiation

determination of symmetry

carbohydrate transport

cell cycle phase

organic acid biosynthetic process

response to virus

regulation of neurological process

regulation of cell organization and biogenesis

innate immune response

negative regulation of enzyme activity

regulation of hydrolase activity

tube morphogenesis

macromolecule biosynthetic process

anatomical structure formation

homophilic cell adhesion

\begin{tabular}{|c|c|}
\hline 0.5792585 & \\
\hline 0.5792585 & \\
\hline 0.5792585 & \\
\hline 0.5792585 & \\
\hline 0.5792585 & \\
\hline 0.5856398 & \\
\hline 0.5928768 & \\
\hline 0.5930421 & \\
\hline 0.6157702 & \\
\hline 0.6157702 & \\
\hline 0.6157702 & \\
\hline 0.6163635 & \\
\hline 0.6196558 & \\
\hline 0.6236839 & \\
\hline 0.6632268 & \\
\hline 0.6632268 & \\
\hline 0.6632268 & \\
\hline 0.6632268 & \\
\hline 0.6661591 & \\
\hline 0.667289 & \\
\hline 0.667289 & \\
\hline 0.6820303 & \\
\hline 0.6820303 & \\
\hline 0.6820303 & \\
\hline 0.7038767 & \\
\hline 0.7090337 & \\
\hline 0.7343629 & \\
\hline 0.7343629 & \\
\hline 0.7372031 & \\
\hline 0.7568814 & \\
\hline 0.7705156 & \\
\hline 0.7772335 & \\
\hline 0.7872186 & \\
\hline 0.800563 & \\
\hline 0.8244722 & \\
\hline 0.8244781 & \\
\hline
\end{tabular}


regulation of cell differentiation

electron transport

establishment of cellular localization

0.8717725

protein transport

peroxisome organization and biogenesis

0.9055007

\section{pregnancy}

sulfur utilization

intercellular junction assembly and maintenance

humoral immune response

energy derivation by oxidation of organic compounds

muscle cell differentiation

DNA metabolic process

regulation of helicase activity

neurotransmitter transport

regulation of lipid metabolic process

transcription

pigment biosynthetic process

two-component signal transduction system (phosphorela

porphyrin metabolic process

detection of hormone stimulus

keratinocyte differentiation

behavioral fear response

polyol transport

maternal behavior

antigen processing and presentation via MHC class Ib

alkene biosynthetic process

mating behavior

mechanoreceptor differentiation

protein complex assembly

esponse to metal ion

imprinting

autophagic vacuole formation

male gamete generation

regulation of growth rate 
positive regulation of developmental process

behavioral response to pain

lymphocyte homeostasis

indole and derivative metabolic process

startle response

polyol metabolic process

regulation of $\mathrm{pH}$

sperm motility

nucleobase, nucleoside, nucleotide and nucleic acid trar

sulfation

taxis

sensory perception of pain

endocrine system development

water homeostasis

viral infectious cycle

morphogenesis of an epithelium

antigen processing and presentation of exogenous antig

regulation of response to biotic stimulus

positive regulation of response to stimulus

positive regulation of gene expression, epigenetic

fluid transport

detection of tumor cell

immune response to tumor cell

nutrient import

hydrogen transport

positive regulation of multicellular organismal process

positive regulation of cytokine production

regulation of embryonic development

nucleoside salvage

vasodilation

regulation of cell redox homeostasis

\begin{tabular}{|c|c|}
\hline 0.23 & 1 \\
\hline 4.64 & 1 \\
\hline 0.23 & 1 \\
\hline 0.7 & 1 \\
\hline 0.93 & 1 \\
\hline 0.7 & 1 \\
\hline 0 & 1 \\
\hline 0 & 1 \\
\hline 0 & 1 \\
\hline 0 & 1 \\
\hline 0.23 & 1 \\
\hline 0.23 & 1 \\
\hline 0.23 & 1 \\
\hline 0.23 & 1 \\
\hline 0 & 1 \\
\hline 1.16 & 1 \\
\hline 0.23 & 1 \\
\hline 0 & 1 \\
\hline 0 & 1 \\
\hline 0.23 & 1 \\
\hline 1.16 & 1 \\
\hline 0 & 1 \\
\hline 0 & 1 \\
\hline 0 & 1 \\
\hline 0 & 1 \\
\hline 0 & 1 \\
\hline 0 & 1 \\
\hline 0 & 1 \\
\hline 0 & 1 \\
\hline 0.23 & 1 \\
\hline 0.93 & 1 \\
\hline 0 & 1 \\
\hline 0 & 1 \\
\hline 0 & 1 \\
\hline 0 & 1 \\
\hline 0 & 1 \\
\hline
\end{tabular}


generation of a signal involved in cell-cell signaling

cofactor catabolic process

phenol metabolic process

myeloid cell homeostasis

organic acid transport

organelle localization

movement in environment of other organism during sym

regulation of organ size

ethanol metabolic process

vitamin transport

regulation of biosynthetic process

aromatic compound catabolic process

purine salvage

hormone catabolic process

negative regulation of gene expression, epigenetic

positive regulation of locomotion

quinone cofactor metabolic process

suckling behavior

cofactor transport

ciliary or flagellar motility

ribosome biogenesis and assembly

cell-matrix adhesion

amino acid derivative metabolic process

cell envelope organization and biogenesis

nucleotide-sugar metabolic process

memory

protein complex disassembly

adaptive immune response

modification of morphology or physiology of other organi

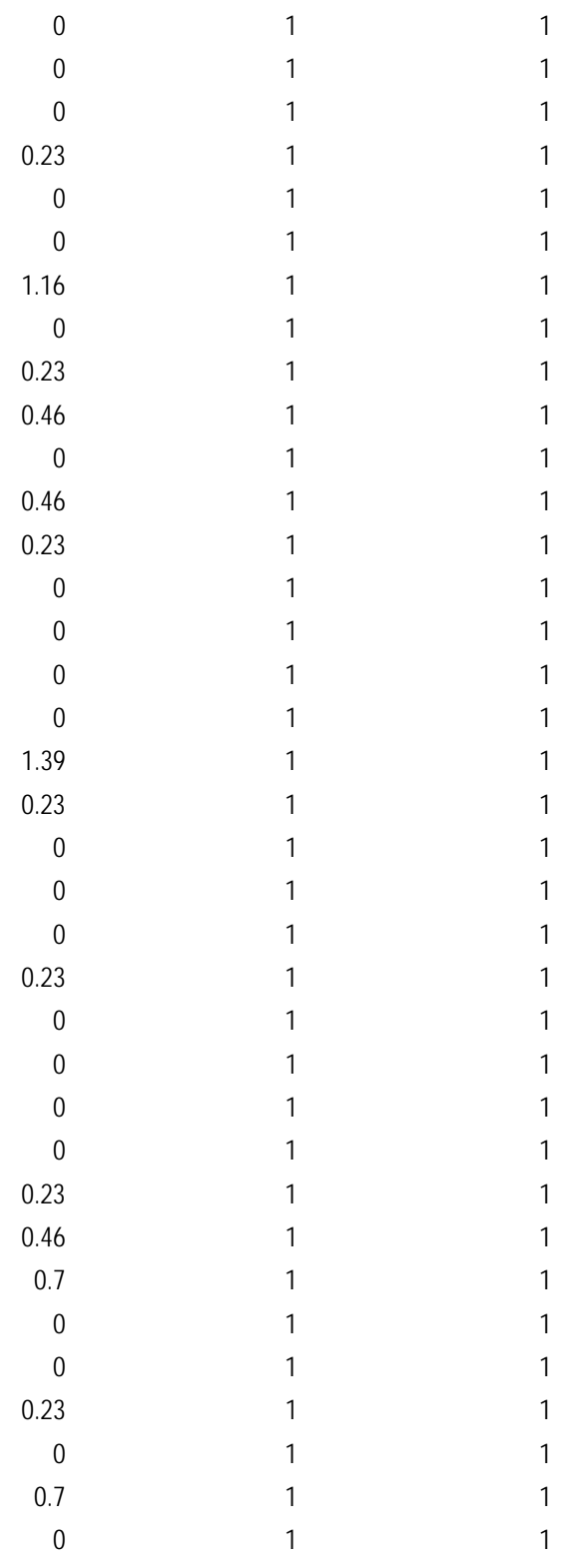


positive regulation of enzyme activity

nitric oxide biosynthetic process

response to X-ray

0.17

cell glucose homeostasis

$\begin{array}{ll}1 & 1 \\ 1 & 1 \\ 1 & 1 \\ 1 & 1\end{array}$


Gene Ontology : biological process. Level: 6 protein modification

sensory perception of smell

G-protein coupled receptor protein signaling pathway

adult walking behavior

phosphorylation

embryonic development (sensu Vertebrata)

visual behavior

visual perception

anterior/posterior pattern formation

DNA strand elongation

negative regulation of bone remodeling

regulation of phospholipase A2 activity

cellular lipid catabolic process

cellular polysaccharide metabolic process

amine biosynthetic process

smoothened signaling pathway

energy reserve metabolic process

regulation of kinase activity

protein oligomerization

defense response to bacterium

sensory organ development

DNA recombination

regulation of cell size

protein catabolic process

modification-dependent macromolecule catabolic proces

detection of light stimulus

defense response to fungus

neuron adhesion

vitamin A metabolic process

synaptic vesicle transport

positive regulation of immune system process

exocytosis

synaptic transmission

kidney development

Wnt receptor signaling pathway
Percentage of genes with allele-specific expression

Percentage of genes without allele-specific expression

Unadjusted pvalue Adjusted pvalueFDR

18.93

1.02

6.93

0

8.86

1.11

0.09

166

0.92

0

0.28

0.28

0.65

0.18

0.18

2.12

0.46

0.46

0.83

0.83

1.94

1.94

1.39

0.09

0.09

0.09

0.09

0.28

1.02

1.11

4.16

0.74

1.39

\begin{tabular}{|c|c|c|}
\hline 11.87 & 0.001320007 & 1 \\
\hline 3.03 & 0.00867637 & 1 \\
\hline 10.86 & 0.01683893 & 1 \\
\hline 0.76 & 0.01908822 & 1 \\
\hline 5.3 & 0.02899869 & 1 \\
\hline 0 & 0.04382327 & 1 \\
\hline 0.76 & 0.06110558 & 1 \\
\hline 3.28 & 0.06460708 & 1 \\
\hline 0 & 0.0709744 & 1 \\
\hline 0.51 & 0.0715566 & 1 \\
\hline 0.51 & 0.0715566 & 1 \\
\hline 0.51 & 0.0715566 & 1 \\
\hline 1.01 & 0.08760815 & 1 \\
\hline 1.01 & 0.08760815 & 1 \\
\hline 1.52 & 0.122405 & 1 \\
\hline 0.76 & 0.12275 & 1 \\
\hline 0.76 & 0.12275 & 1 \\
\hline 3.54 & 0.1336111 & 1 \\
\hline 1.26 & 0.1436778 & 1 \\
\hline 1.26 & 0.1436778 & 1 \\
\hline 1.77 & 0.1531851 & 1 \\
\hline 1.77 & 0.1531851 & 1 \\
\hline 0.76 & 0.1609293 & 1 \\
\hline 3.28 & 0.1678934 & 1 \\
\hline 2.53 & 0.1689792 & 1 \\
\hline 0.51 & 0.1764934 & 1 \\
\hline 0.51 & 0.1764934 & 1 \\
\hline 0.51 & 0.1764934 & 1 \\
\hline 0.51 & 0.1764934 & 1 \\
\hline 0.76 & 0.1980979 & 1 \\
\hline 0.25 & 0.1995551 & 1 \\
\hline 2.02 & 0.2034832 & 1 \\
\hline 5.81 & 0.2062397 & 1 \\
\hline 1.52 & 0.2214429 & 1 \\
\hline 2.27 & 0.2470893 & 1 \\
\hline
\end{tabular}


positive regulation of cell differentiation

Notch signaling pathway

cyclic nucleotide metabolic process

regulation of cell migration

glutamate signaling pathway

biopolymer glycosylation

mRNA metabolic process

carbohydrate mediated signaling

locomotor rhythm

positive regulation of bone remodeling

S-adenosylmethionine metabolic process

protein insertion into membrane

baroreceptor feedback regulation of blood pressure

circadian sleep/wake cycle

negative regulation of carbohydrate metabolic process

regulation of nitric oxide biosynthetic process

peptide modification

positive regulation of catabolic process

polysaccharide catabolic process

response to cocaine

carboxylic acid catabolic process

regulation of blood pressure by hormones

superoxide release

behavioral response to ethano

amino acid derivative catabolic process

sensory perception of taste

regulation of cellular catabolic process

phospholipid scrambling

response to reactive oxygen species

response to morphine

muscle cell development

centrosome organization and biogenesis

antibiotic biosynthetic process

detection of bacterium

negative regulation of catabolic process

DNA geometric change

0.261897

0.2631478

0.2677485

0.2677485

0.2677485

0.2677485

0.2677485

0.2677485

0.2677485

0.2677485

0.2677485

0.2677485

0.2677485

0.2677485

0.2677485

0.2677485

0.2677485

0.2677485

0.2677485

0.2677485

0.2677485

0.2677485

0.2677485

0.2677485

0.2677485

0.2677485

0.2677485

0.2677485

0.2677485

0.2677485

0.2677485 
regulation of vasoconstriction

nitric oxide mediated signal transduction

substrate-bound cell migration

cell fate determination

cell-cell signaling during cell fate commitment

0.2713158

0.2918811

0.2918811

0.2918811

glycerolipid metabolic process

mesenchymal cell development

biopolymer biosynthetic process

peptidoglycan metabolic process

neutral lipid metabolic process

female sex differentiation

monosaccharide metabolic process

skeletal development

central nervous system development

0.2918811

0.2918811

0.2918811

0.2918811

0.2918811

0.2918811

0.3124353

0.3202762

0.3297796

oxidoreduction coenzyme metabolic process

0.351682

0.3538307

0.3538307

anion transport

positive regulation of cellular metabolic process

0.3638177

0.3761687

regulation of immune response

0.3761687

0.379194

0.379194

0.379194

coenzyme biosynthetic process

negative regulation of signal transduction

0.3932599

0.4150825

cell death

glycoprotein metabolic process

0.4168532

0.4168532

0.4171678

0.4290147

0.438683

0.4480905

0.4480905

0.4480905 
pteridine and derivative biosynthetic process

peptide secretion

negative regulation of cell motility

regulation of smooth muscle contraction

positive regulation of heart contraction

molybdopterin cofactor metabolic process

positive regulation of carbohydrate metabolic process

ovulation

regulation of nitric-oxide synthase activity

natural killer cell activation

porphyrin catabolic process

leukocyte activation during immune response

arachidonic acid secretion

plasma membrane repair

oligopeptide transport

intestinal absorption

morphogenesis of an epithelial shee

ipoprotein metabolic process

acute inflammatory response

positive regulation of transferase activity

branching morphogenesis of a tube

positive regulation of biosynthetic process

regulation of cell morphogenesis

neurogenesis

actin filament-based process

cell projection morphogenesis

limb morphogenesis

embryonic appendage morphogenesis

microtubule-based process

ytokine metabolic process

regulation of fat cell differentiation

response to UV

\begin{tabular}{|c|c|}
\hline 0.4556657 & \\
\hline 0.4586838 & \\
\hline 0.4639404 & \\
\hline 0.4639404 & \\
\hline 0.4639404 & \\
\hline 0.4639404 & \\
\hline 0.4639404 & \\
\hline 0.4639404 & \\
\hline 0.4639404 & \\
\hline 0.4639404 & \\
\hline 0.4639404 & \\
\hline 0.4639404 & \\
\hline 0.4639404 & \\
\hline 0.4639404 & \\
\hline 0.4639404 & \\
\hline 0.4639404 & \\
\hline 0.4639404 & \\
\hline 0.4639404 & \\
\hline 0.4639404 & \\
\hline 0.4639404 & \\
\hline 0.4639404 & \\
\hline 0.4718883 & \\
\hline 0.4718883 & \\
\hline 0.4873266 & \\
\hline 0.4982371 & \\
\hline 0.4982371 & \\
\hline 0.4982371 & \\
\hline 0.5025364 & \\
\hline 0.5030137 & \\
\hline 0.5030137 & \\
\hline 0.5323865 & \\
\hline 0.5323865 & \\
\hline 0.5325448 & \\
\hline 0.56311 & \\
\hline 0.5688295 & \\
\hline 0.5688295 & \\
\hline
\end{tabular}


cortical cytoskeleton organization and biogenesis $\quad 0.28$

acetyl-CoA metabolic process $\quad 0.28$

leukocyte adhesion

RNA modification

positive regulation of cell organization and biogenesis

nucleobase biosynthetic process

nucleoside monophosphate metabolic process

regulation of inflammatory response

germ cell migration

negative regulation of cyclase activity

negative regulation of lyase activity

immune response-regulating cell surface receptor signal

regulation of protein secretion

positive regulation of secretion

water-soluble vitamin biosynthetic process

cellular respiration

protein import

vasculature development

heart developmen

cellular morphogenesis during differentiation

amino acid derivative biosynthetic process

proteoglycan metabolic process

negative regulation of cell organization and biogenesis

regulation of epithelial cell proliferation

lysosome organization and biogenesis

protein depolymerization

cytokine and chemokine mediated signaling pathway

protein folding

DNA replication

small GTPase mediated signal transduction

regulation of nucleobase, nucleoside, nucleotide and nu

regulation of action potential

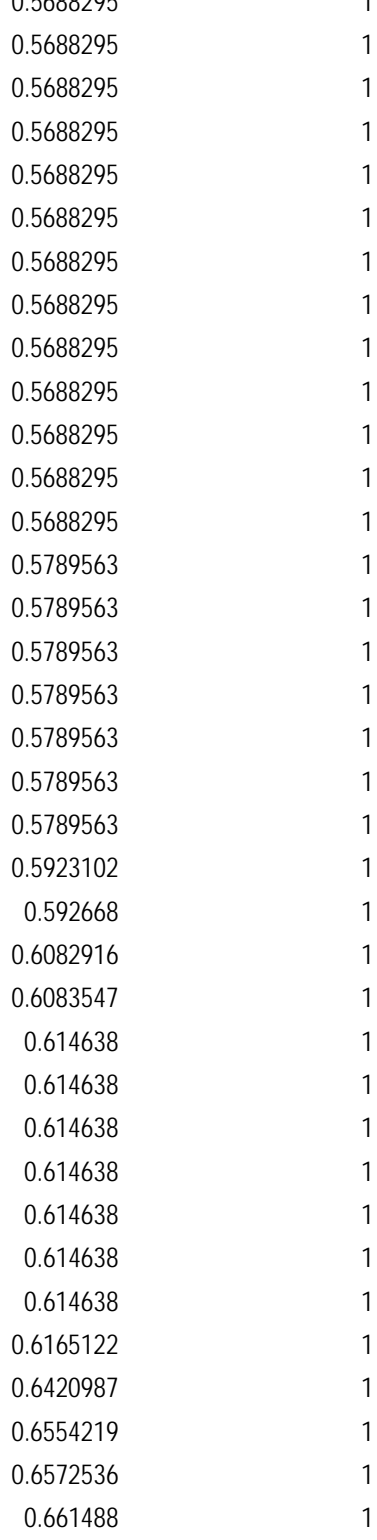




\begin{tabular}{|c|c|c|c|}
\hline 37 & 0.51 & 0.661488 & 1 \\
\hline 37 & 0.51 & 0.661488 & 1 \\
\hline 52 & 3.79 & 0.6650541 & 1 \\
\hline 4 & 1.52 & 0.6676904 & 1 \\
\hline 85 & 2.27 & 0.6717916 & 1 \\
\hline 5 & 0.25 & 0.6822859 & 1 \\
\hline 55 & 0.25 & 0.6822859 & 1 \\
\hline 5 & 0.25 & 0.6822859 & 1 \\
\hline 22 & 1.77 & 0.6859411 & 1 \\
\hline 5 & 0.25 & 0.6897683 & 1 \\
\hline 65 & 0.25 & 0.6897683 & 1 \\
\hline 65 & 0.25 & 0.6897683 & 1 \\
\hline 54 & 6.06 & 0.7042588 & . \\
\hline 55 & 0.76 & 0.7079658 & 1 \\
\hline 55 & 0.76 & 0.7079658 & 1 \\
\hline 55 & 0.76 & 0.7079658 & 1 \\
\hline 72 & 6.31 & 0.7081859 & 1 \\
\hline 82 & 6.31 & 0.7107259 & 1 \\
\hline 49 & 2.78 & 0.7144068 & 1 \\
\hline 19 & 2.78 & 0.7144068 & 1 \\
\hline 77 & 2.27 & 0.7152497 & 1 \\
\hline ; & 0.76 & 0.7327987 & 1 \\
\hline 65 & 0.76 & 0.7327987 & 1 \\
\hline 33 & 0.51 & 0.7372247 & 1 \\
\hline 83 & 0.51 & 0.7372247 & 1 \\
\hline 4 & 1.01 & 0.7434302 & 1 \\
\hline 83 & 1.01 & 0.7558127 & 1 \\
\hline 33 & 1.01 & 0.7558127 & 1 \\
\hline 33 & 1.01 & 0.7558127 & 1 \\
\hline 33 & 1.01 & 0.7558127 & 1 \\
\hline 0 & 1.26 & 0.7765899 & 1 \\
\hline$J 2$ & 1.26 & 0.7765899 & 1 \\
\hline 29 & 1.01 & 0.7935596 & 1 \\
\hline ) & 1.26 & 0.8107554 & 1 \\
\hline 75 & 2.02 & 0.8263799 & 1 \\
\hline 3 & 2.02 & 0.8305251 & . \\
\hline
\end{tabular}

\section{protein kinase cascade}

$M$ phase

lipid biosynthetic process

segmentation

determination of bilateral symmetry

positive regulation of transport

DNA packaging

germ cell development

group transfer coenzyme metabolic process

epithelial cell differentiation

cation transport

carboxylic acid biosynthetic process

cellular protein complex assembly

development of primary sexual characteristics

regulation of progression through cell cycle

intracellular transport

muscle development

positive regulation of cell proliferation

chromosome organization and biogenesis (sensu Eukar

positive regulation of hydrolase activity

negative regulation of protein metabolic process

ribonucleotide metabolic process

biogenic amine metabolic process

leukocyte mediated immunity

cell maturation

antimicrobial humoral response

regulation of lymphocyte activation

negative regulation of cell differentiation

carbohydrate biosynthetic process

regulated secretory pathway

steroid metabolic process

amino acid metabolic process 
mast cell activation

positive regulation of cyclase activity

benzene and derivative metabolic process

regulation of pigment cell differentiation

hemoglobin metabolic process

negative regulation of helicase activity

deoxyribonucleoside metabolic process

uroporphyrinogen III metabolic process

ameboidal cell migration

modification of host morphology or physiology

immune system development

positive regulation of protein metabolic process

oogenesis

amino sugar metabolic process

regulation of smooth muscle cell proliferation

negative regulation of heart contraction

morphogenesis of a polarized epithelium

spermatogenesis

stress-activated protein kinase signaling pathway

vitamin D metabolic process

purine nucleoside metabolic process

negative regulation of neurological process

DNA catabolic process

regulation of muscle cell differentiation

embryonic axis specification

DNA damage response, signal transduction by p53 clas:

vesicle fusion

cellular carbohydrate catabolic process

formation of immunological synapse

cell fate specification

DNA repair

pyrimidine base metabolic process 
melanocyte differentiation

positive regulation of lipid metabolic process

nucleotide-sugar biosynthetic process

nucleic acid transport

negative regulation of secretion

lipid raft organization and biogenesis

dorsal/ventral pattern formation

mitochondrial membrane organization and biogenesis

phosphoenolpyruvate-dependent sugar phosphotransfer

MHC class II biosynthetic process

proximal/distal pattern formation

positive regulation of lyase activity

negative regulation of defense response

nucleotide-sugar transport

regulation of cellular biosynthetic process

viral genome replication

positive regulation of cell motility

daptive immune response (sensu Gnathostomata)

disruption of cells of other organism during symbiotic int

detection of fungus

response to mercury ion

catecholamine transport

long-term memory

nucleus localization

ER-nuclear signaling pathway

glycerol metabolic process

RNA metabolic process

mitochondrial genome maintenance

ndodermal cell fate commitmen

morphogenesis of embryonic epithelium

nucleoside catabolic process 
production of molecular mediator of immune response

neutrophil homeostasis

T cell homeostasis

defense response to virus

biopolymer methylation

purine base metabolic process

second-messenger-mediated signaling

posttranscriptional gene silencing

dopamine transport

establishment of organelle localization

maintenance of DNA repeat elements

cytoskeletal anchoring

ribonucleoside metabolic process

endothelial cell migration

thiamin and derivative metabolic process

RNA catabolic process

entry into other organism during symbiotic interaction

nucleobase catabolic process

protein polymerization

negative regulation of biosynthetic process

water transport

regulation of fibroblast proliferation

regulation of vasodilation

peripheral nervous system development

anion homeostasis

embryonic organ development

choline transport

lymphocyte proliferation

regulation of GTPase activity

regulation of response to tumor cell

negative regulation of transport

\begin{tabular}{|c|c|c|}
\hline 0.51 & 1 & 1 \\
\hline 0.25 & 1 & 1 \\
\hline 0 & 1 & 1 \\
\hline 0.25 & 1 & 1 \\
\hline 0.25 & 1 & 1 \\
\hline 0.51 & 1 & 1 \\
\hline 0 & 1 & 1 \\
\hline 0 & 1 & 1 \\
\hline 0 & 1 & 1 \\
\hline 0 & 1 & 1 \\
\hline 0 & 1 & 1 \\
\hline 2.78 & 1 & 1 \\
\hline 0 & 1 & 1 \\
\hline 0 & 1 & 1 \\
\hline 0.25 & 1 & 1 \\
\hline 0 & 1 & 1 \\
\hline 0 & 1 & 1 \\
\hline 0 & 1 & 1 \\
\hline 0 & 1 & 1 \\
\hline 0 & 1 & 1 \\
\hline 0 & 1 & 1 \\
\hline 0 & 1 & 1 \\
\hline 0 & 1 & 1 \\
\hline 0.51 & 1 & 1 \\
\hline 0.25 & 1 & 1 \\
\hline 0 & 1 & 1 \\
\hline 0.25 & 1 & 1 \\
\hline 0 & 1 & 1 \\
\hline 0.25 & 1 & 1 \\
\hline 0 & 1 & 1 \\
\hline 0.25 & 1 & 1 \\
\hline 0 & 1 & 1 \\
\hline 0.76 & 1 & 1 \\
\hline 0.51 & 1 & 1 \\
\hline 0 & 1 & 1 \\
\hline 0.25 & 1 & 1 \\
\hline
\end{tabular}


regulation of sensory perception

hormone-mediated signaling

associative learning

isoprenoid metabolic process

neural tube development

regulation of protein stability

nuclear body organization and biogenesis

positive regulation of defense response

regulation of phosphorus metabolic process

protein maturation

initiation of viral infection

intercellular junction assembly

positive regulation of signal transduction

monoamine transport

chromosome condensation

$\mathrm{pH}$ reduction

gland development

male sex differentiation

indole derivative metabolic process

oxygen transport

sterol transport

pancreas development

negative regulation of cell proliferation

enzyme linked receptor protein signaling pathway

carboxylic acid transport

nucleoside diphosphate metabolic process

brown fat cell differentiation

heme metabolic process

modification of structure of other organism during symbi

smooth muscle cell differentiation

vitamin B6 metabolic process

cation homeostasis

\begin{tabular}{|c|c|c|}
\hline 1.26 & 1 & 1 \\
\hline 0.25 & 1 & 1 \\
\hline 0 & 1 & 1 \\
\hline 0 & 1 & 1 \\
\hline 0 & 1 & 1 \\
\hline 0 & 1 & 1 \\
\hline 0.25 & 1 & 1 \\
\hline 0.25 & 1 & 1 \\
\hline 0.25 & 1 & 1 \\
\hline 0 & 1 & 1 \\
\hline 0 & 1 & 1 \\
\hline 0 & 1 & 1 \\
\hline 0.51 & 1 & 1 \\
\hline 0 & 1 & 1 \\
\hline 0 & 1 & 1 \\
\hline 0.25 & 1 & 1 \\
\hline 1.26 & 1 & 1 \\
\hline 0 & 1 & 1 \\
\hline 0.25 & 1 & 1 \\
\hline 0 & 1 & 1 \\
\hline 0.25 & 1 & 1 \\
\hline 0.25 & 1 & 1 \\
\hline 0 & 1 & 1 \\
\hline 0 & 1 & 1 \\
\hline 0 & 1 & 1 \\
\hline 0 & 1 & 1 \\
\hline 2.78 & 1 & 1 \\
\hline 3.79 & 1 & 1 \\
\hline 0.51 & 1 & 1 \\
\hline 0 & 1 & 1 \\
\hline 0 & 1 & 1 \\
\hline 0 & 1 & 1 \\
\hline 0 & 1 & 1 \\
\hline 0 & 1 & 1 \\
\hline 0 & 1 & 1 \\
\hline 1.52 & 1 & 1 \\
\hline
\end{tabular}


intermediate filament-based process

\section{gut development}

cellular protein complex disassembly

nucleotide catabolic process

negative regulation of organ size

sulfate assimilation

negative regulation of cell adhesion

lipid raft localization

aminophospholipid transport

leukocyte migration

embryo implantation

regulation of protein complex assembly

proteolysis

integrin-mediated signaling pathway

nucleotide biosynthetic process

nucleobase transport

porphyrin biosynthetic process

response to starvation

regulation of endothelial cell proliferation

regulation of immune effector process

regulation of protein transport

interphase

pyrimidine nucleotide metabolic process

vesicle localization

coenzyme catabolic process

vesicle docking

B cell homeostasis

menstrual cycle process

genitalia developmen

antigen processing and presentation of lipid antigen via 
ethanol oxidation

RNA-mediated gene silencing

monosaccharide transport

0


Gene Ontology : biological process. Level: 7 protein homooligomerization

biomineral formation

double-strand break repair

\section{visual learning}

cellular protein catabolic process

heparan sulfate proteoglycan metabolic process

adult heart development

epithelial to mesenchymal transition

calcium ion-dependent exocytosis

recombinational repair

positive regulation of phospholipase A2 activity

protein amino acid phosphorylation

regulation of protein kinase activity

intracellular protein transport

membrane lipid catabolic process

cyclic nucleotide biosynthetic process

acute-phase response

inorganic anion transport

DNA-dependent DNA replication

muscle fiber development

positive regulation of epithelial cell proliferation

polysaccharide biosynthetic process

detection of visible light

detection of light stimulus during sensory perception

endoderm development

fatty acid metabolic process

positive regulation of nucleobase, nucleoside, nucleotide

cGMP metabolic process

glycolipid metabolic process

embryonic development (sensu Mammalia)

amino acid biosynthetic process

ear development

cell growth

mRNA processing

peptidoglycan catabolic process
Percentage of genes with allele-specific expression 0.11

Percentage of genes without allele-specific expression

Unadjusted pvalue Adjusted pvalueFDR

0.76

0.1

0.11
1.95

0

0

0

0

0
9.87

2.28

3.69

0.22

0.22

0.22

1.19

0.65

0.65

0.11

0.11

0.11

0.11

0.11

1.74

4.34

0.33

0.33

0.76

0.43

0.43

2.17

2.17

$\begin{array}{rl}0.005359601 & 1 \\ 0.009100373 & 1 \\ 0.01759404 & 1 \\ 0.05560903 & 1 \\ 0.05866208 & 1 \\ 0.06684507 & 1 \\ 0.06684507 & 1 \\ 0.06684507 & 1 \\ 0.06684507 & 1 \\ 0.06684507 & 1 \\ 0.06684507 & 1 \\ 0.07142957 & 1 \\ 0.07548919 & 1 \\ 0.07890425 & 1 \\ 0.1126315 & 1 \\ 0.1126315 & 1 \\ 0.1126315 & 1 \\ 0.1153819 & 1 \\ 0.1648446 & 1 \\ 0.1648446 & 1 \\ 0.1660901 & 1 \\ 0.1660901 & 1 \\ 0.1660901 & 1 \\ 0.1660901 & 1 \\ 0.1660901 & 1 \\ 0.1721741 & 1 \\ 0.17787 & 1 \\ 0.1832001 & 1 \\ 0.1832001 & 1 \\ 0.2005548 & 1 \\ 0.2160468 & 1 \\ 0.2160468 & 1 \\ 0.22816 & 1 \\ 0.22816 & 1 \\ 0.2588424 & 1 \\ & 1 \\ & 1 \\ 0 & 1 \\ 0 & 1 \\ 0 & 1 \\ 0 & 1 \\ 0 & 1 \\ 0 & 1 \\ 0 & 1\end{array}$


lipid phosphorylation

baroreceptor response to lowering of blood pressure

0.2588424

0.2588424

0.2588424

0.2588424

bile acid metabolic process

peroxisomal transport

negative regulation of cellular catabolic process

biogenic amine catabolic process

cellular extravasation

actin filament severing

positive regulation of vasoconstriction

positive regulation of cellular catabolic process

defense response to Gram-positive bacterium

receptor guanylyl cyclase signaling pathway

prostate gland development

cellular polysaccharide catabolic process

regulation of Notch signaling pathway

peptide amidation

salivary gland development

centrosome cycle

antibacterial humoral response

leading edge cell differentiation

metabotropic glutamate receptor signaling pathway

DNA duplex unwinding

antifungal humoral response

mitotic recombination

bilirubin conjugation

non-recombinational repair

positive regulation of nitric oxide biosynthetic process

response to hydrogen peroxid

norepinephrine-epinephrine regulation of blood pressure

ER overload response

0.2588424

0.2588424

0.2588424

0.2588424

0.2588424

0.2588424

0.2588424

0.2588424

0.2588424

0.2588424

0.2588424

0.2588424

0.2588424

0.2588424

0.2588424

0.2588424

0.2588424

0.2588424

0.2588424

0.2588424

0.2588424

0.2588424

0.2588424

0.2588424

0.2588424

0.2588424

0.2588424

0.2588424 
extracellular polysaccharide metabolic process

regulation of urothelial cell proliferation

\begin{tabular}{|c|c|}
\hline 0.31 & 0.2588424 \\
\hline 0.31 & 0.2588424 \\
\hline 0.31 & 0.2588424 \\
\hline 1.24 & 0.2636428 \\
\hline 0.62 & 0.2765711 \\
\hline 0.62 & 0.2765711 \\
\hline 0.62 & 0.2765711 \\
\hline 0.62 & 0.2765711 \\
\hline 0.62 & 0.2765711 \\
\hline 0.62 & 0.2765711 \\
\hline 0.62 & 0.2765711 \\
\hline 0.62 & 0.2765711 \\
\hline 0.62 & 0.2765711 \\
\hline 0.62 & 0.2765711 \\
\hline 0.62 & 0.2765711 \\
\hline 0.62 & 0.2765711 \\
\hline 4.35 & 0.2825478 \\
\hline 3.11 & 0.2871908 \\
\hline 0.93 & 0.315395 \\
\hline 0.93 & 0.315395 \\
\hline 0.31 & 0.3167569 \\
\hline 0.31 & 0.3167569 \\
\hline 6.83 & 0.3291208 \\
\hline 3.11 & 0.3317314 \\
\hline 0 & 0.3356444 \\
\hline 0 & 0.3356444 \\
\hline 6.52 & 0.3371278 \\
\hline 1.55 & 0.3393898 \\
\hline 0.93 & 0.383902 \\
\hline 0.93 & 0.383902 \\
\hline 0.93 & 0.383902 \\
\hline 3.11 & 0.3977976 \\
\hline 3.42 & 0.4273414 \\
\hline 0.93 & 0.4337513 \\
\hline 0.93 & 0.4337513 \\
\hline 0.93 & 0.4337513 \\
\hline
\end{tabular}

ycoprotein biosynthetic process

dicarboxylic acid transport

transmembrane receptor protein tyrosine phosphatase s

glucan metabolic process

development of primary female sexual characteristics

induction

glycoprotein catabolic process

acylglycerol metabolic process

frizzled signaling pathway

photoreceptor cell development

glutamine family amino acid metabolic process

proteoglycan biosynthetic process

aminoglycan biosynthetic process

negative regulation of progression through cell cycle

brain development

ectoderm development

hexose metabolic process

microtubule cytoskeleton organization and biogenesis

positive regulation of immune response

metal ion transport

ubiquitin cycle

dopamine receptor signaling pathway

pyridine nucleotide metabolic process

programmed cell death

phospholipid metabolic process

glycosaminoglycan metabolic process

gonad development

gliogenesis

di-, tri-valent inorganic cation transport

negative regulation of nucleobase, nucleoside, nucleotid

eye development

calcium-mediated signaling

regulation of synaptic transmission 
regulation of smoothened signaling pathway

polyisoprenoid metabolic process

lymphocyte activation during immune response

myeloid leukocyte mediated immunity

regulation of macrophage activation

glycerolipid biosynthetic process

neutral lipid biosynthetic process

Mo-molybdopterin cofactor metabolic process

blastoderm segmentation

leukocyte degranulation

formation of primary germ layer

positive regulation of fibroblast proliferation

cardiac cell differentiation

sulfur amino acid metabolic process

molybdopterin cofactor biosynthetic process

neuromuscular synaptic transmission

positive regulation of cell migration

regulation of RNA metabolic process

lipoprotein catabolic process

retinoid metabolic process

retinoic acid metabolic process

lipopolysaccharide metabolic process

peptide hormone secretion

nuclear transport

steroid hormone receptor signaling pathway

monosaccharide catabolic process

nucleocytoplasmic transport

\begin{tabular}{|c|c|}
\hline 0.93 & 0.4337513 \\
\hline 0.31 & 0.4508398 \\
\hline 0.31 & 0.4508398 \\
\hline 0.31 & 0.4508398 \\
\hline 0.31 & 0.4508398 \\
\hline 0.31 & 0.4508398 \\
\hline 0.31 & 0.4508398 \\
\hline 0.31 & 0.4508398 \\
\hline 0.31 & 0.4508398 \\
\hline 0.31 & 0.4508398 \\
\hline 0.31 & 0.4508398 \\
\hline 0.31 & 0.4508398 \\
\hline 0.31 & 0.4508398 \\
\hline 0.31 & 0.4508398 \\
\hline 0.31 & 0.4508398 \\
\hline 0.31 & 0.4508398 \\
\hline 0.31 & 0.4508398 \\
\hline 0.31 & 0.4508398 \\
\hline 0.31 & 0.4508398 \\
\hline 0.31 & 0.4508398 \\
\hline 0.31 & 0.4508398 \\
\hline 0.31 & 0.4508398 \\
\hline 0.31 & 0.4508398 \\
\hline 0.31 & 0.4508398 \\
\hline 0.31 & 0.4508398 \\
\hline 0.31 & 0.4508398 \\
\hline 0.31 & 0.4508398 \\
\hline 0.31 & 0.4508398 \\
\hline 0.31 & 0.4508398 \\
\hline 2.17 & 0.4528719 \\
\hline 0.31 & 0.4605045 \\
\hline 0.31 & 0.4680323 \\
\hline 2.17 & 0.4732204 \\
\hline 4.66 & 0.482285 \\
\hline 24.22 & 0.4872453 \\
\hline 1.24 & 0.4890871 \\
\hline
\end{tabular}


regulation of $\mathrm{T}$ cell activation

metanephros development

phosphoinositide-mediated signaling

Ras protein signal transduction

regulation of epithelial cell differentiation

aerobic respiration

positive regulation of protein secretion

regulation of chemotaxis

positive chemotaxis

organic anion transport

pinocytosis

positive regulation of progression through cell cycle

serine family amino acid metabolic process

pyruvate metabolic process

unfolded protein response

folic acid and derivative metabolic process

monovalent inorganic cation homeostasis

somitogenesis

monosaccharide biosynthetic process

peptide cross-linking

regulation of cytokine secretion

oligosaccharide biosynthetic process

amino acid activation

gastrulation (sensu Deuterostomia)

oxidative phosphorylation

protein tetramerization

ribonucleoside monophosphate metabolic process

nucleoside monophosphate biosynthetic process

mitochondrial transport

regulation of Wnt receptor signaling pathway

negative regulation of cell size

base-excision repair

protein amino acid ADP-ribosylation

nucleotide-excision repair

biogenic amine biosynthetic process

ensheathment of neurons

\begin{tabular}{|c|c|}
\hline 1.24 & 0.4890871 \\
\hline 1.24 & 0.4890871 \\
\hline 1.55 & 0.5539047 \\
\hline 2.17 & 0.5560774 \\
\hline 0 & 0.5728754 \\
\hline 0 & 0.5728754 \\
\hline 0 & 0.5728754 \\
\hline 0 & 0.5728754 \\
\hline 0 & 0.5728754 \\
\hline 0 & 0.5728754 \\
\hline 0 & 0.5728754 \\
\hline 0 & 0.5728754 \\
\hline 0 & 0.5728754 \\
\hline 0 & 0.5728754 \\
\hline 0 & 0.5728754 \\
\hline 0 & 0.5728754 \\
\hline 0 & 0.5728754 \\
\hline 0 & 0.5728754 \\
\hline 0 & 0.5728754 \\
\hline 0 & 0.5728754 \\
\hline 0 & 0.5728754 \\
\hline 0 & 0.5728754 \\
\hline 0 & 0.5728754 \\
\hline 0 & 0.5728754 \\
\hline 0 & 0.5728754 \\
\hline 0 & 0.5728754 \\
\hline 0 & 0.5728754 \\
\hline 0 & 0.5728754 \\
\hline 0 & 0.5778086 \\
\hline 0 & 0.5778086 \\
\hline 0 & 0.5778086 \\
\hline 0 & 0.5778086 \\
\hline 0 & 0.5778086 \\
\hline 0 & 0.5778086 \\
\hline 0.62 & 0.6088771 \\
\hline 0.62 & 0.6088771 \\
\hline
\end{tabular}


protein amino acid dephosphorylation

translational initiation

amino acid catabolic process

mesoderm development

steroid biosynthetic process

cell cycle checkpoint

determination of leftright symmetry

cartilage development

regulation of DNA metabolic process

leukocyte chemotaxis

hemopoietic or lymphoid organ development

blood vessel development

membrane lipid biosynthetic process

positive regulation of cellular biosynthetic process

actin cytoskeleton organization and biogenesis

ribonucleotide biosynthetic process

transmembrane receptor protein serine/threonine kinase

embryonic limb morphogenesis

antimicrobial humoral response (sensu Vertebrata)

transcription, DNA-dependent

cytokine biosynthetic process

neuropeptide signaling pathway

striated muscle development

di-, tri-valent inorganic cation homeostasis

cyclic-nucleotide-mediated signaling

establishment and/or maintenance of chromatin architec

rransmembrane receptor protein tyrosine kinase signalin

tRNA processing

synaptic vesicle endocytosis

JAK-STAT cascade

pyrimidine ribonucleotide metabolic process

\begin{tabular}{|c|c|}
\hline 0.62 & 0.6088771 \\
\hline 0.62 & 0.6088771 \\
\hline 0.62 & 0.6088771 \\
\hline 3.11 & 0.610386 \\
\hline 1.24 & 0.6229159 \\
\hline 2.48 & 0.6505829 \\
\hline 0.62 & 0.6522511 \\
\hline 0.62 & 0.6522511 \\
\hline 0.62 & 0.6522511 \\
\hline 0.62 & 0.6522511 \\
\hline 0.31 & 0.6844136 \\
\hline 0.31 & 0.6844136 \\
\hline 0.31 & 0.6844136 \\
\hline 0.31 & 0.6844136 \\
\hline 0.31 & 0.6880704 \\
\hline 3.42 & 0.7128128 \\
\hline 2.8 & 0.7159592 \\
\hline 0.93 & 0.7247405 \\
\hline 0.93 & 0.7247405 \\
\hline 3.11 & 0.7288196 \\
\hline 0.62 & 0.738273 \\
\hline 0.62 & 0.7413155 \\
\hline 0.62 & 0.7413155 \\
\hline 1.24 & 0.7507852 \\
\hline 22.67 & 0.7544534 \\
\hline 1.24 & 0.7650119 \\
\hline 1.55 & 0.7811147 \\
\hline 1.86 & 0.8024785 \\
\hline 1.86 & 0.8106393 \\
\hline 1.55 & 0.8116982 \\
\hline 2.17 & 0.8360549 \\
\hline 3.11 & 0.85016 \\
\hline 0.31 & 1 \\
\hline 0 & 1 \\
\hline 0.31 & 1 \\
\hline 0.31 & 1 \\
\hline
\end{tabular}


regulation of melanocyte differentiation

mRNA catabolic process

monovalent inorganic cation transport

posttranslational protein folding

pyridoxine metabolic process

mRNA modification

lipid raft distribution

mesonephros development

pyrimidine base biosynthetic process

MAPKKK cascade

heme oxidation

mammary gland development

hexose transport

pyrimidine nucleotide biosynthetic process

chemokine metabolic process

thiamin metabolic process

regulation of G-protein coupled receptor protein signalin

regulation of translation

positive regulation of smooth muscle cell proliferation

Golgi vesicle transport

negative regulation of cellular biosynthetic process

ubiquinone metabolic process

odontogenesis

$M$ phase of meiotic cell cycle

negative regulation of fibroblast proliferation

interphase of mitotic cell cycle

glycerol-3-phosphate metabolic process

G2 phase

entry into host

negative regulation of epithelial cell proliferation

regulation of sensory perception of pain

I-kappaB kinase/NF-kappaB cascade

\begin{tabular}{|c|c|c|}
\hline 0 & 1 & 1 \\
\hline 0.31 & 1 & 1 \\
\hline 0 & 1 & 1 \\
\hline 0.62 & 1 & 1 \\
\hline 0 & 1 & 1 \\
\hline 0 & 1 & 1 \\
\hline 3.73 & 1 & 1 \\
\hline 0 & 1 & 1 \\
\hline 0 & 1 & 1 \\
\hline 0 & 1 & 1 \\
\hline 0 & 1 & 1 \\
\hline 0 & 1 & 1 \\
\hline 0 & 1 & 1 \\
\hline 1.86 & 1 & 1 \\
\hline 0 & 1 & 1 \\
\hline 0 & 1 & 1 \\
\hline 0.31 & 1 & 1 \\
\hline 0.31 & 1 & 1 \\
\hline 0 & 1 & 1 \\
\hline 0 & 1 & 1 \\
\hline 0.31 & 1 & 1 \\
\hline 1.55 & 1 & 1 \\
\hline 0 & 1 & 1 \\
\hline 0.62 & 1 & 1 \\
\hline 0.31 & 1 & 1 \\
\hline 0 & 1 & 1 \\
\hline 0.31 & 1 & 1 \\
\hline 0.62 & 1 & 1 \\
\hline 0 & 1 & 1 \\
\hline 0.62 & 1 & 1 \\
\hline 0 & 1 & 1 \\
\hline 0 & 1 & 1 \\
\hline 0 & 1 & 1 \\
\hline 0 & 1 & 1 \\
\hline 0 & 1 & 1 \\
\hline 1.86 & 1 & 1 \\
\hline
\end{tabular}


cytoskeleton-dependent intracellular transport

regulation of progression through mitotic cell cycle

pyrimidine nucleoside triphosphate metabolic process

embryonic epithelial tube formation

embryonic skeletal development

single strand break repair

regulation of antiviral response by host

protein amino acid alkylation

protein processing

regulation of neurotransmitter levels

protein amino acid deacetylation

pyrimidine transport

positive regulation of protein complex assembly

nucleoside triphosphate biosynthetic process

thyroid hormone metabolic process

regulation of innate immune response

regulation of protein modification

ribonucleoside diphosphate metabolic process

pyrimidine nucleoside catabolic process

cell killing

mitochondrial DNA metabolic process

ethanolamine and derivative metabolic process

C21-steroid hormone metabolic process

alpha-beta $T$ cell activation

negative regulation of fat cell differentiation

entry into cell of other organism during symbiotic interac

leg morphogenesis

hemoglobin biosynthetic process

RNA-mediated posttranscriptional gene silencing

metal ion homeostasis 
adrenal gland developmen

hindlimb morphogenesis

regulation of exocytosis

positive regulation of fat cell differentiation

serotonin receptor signaling pathway

purine nucleoside diphosphate metabolic process

receptor-mediated endocytosis

positive regulation of immune effector process

pentose metabolic process

protein amino acid acylation

regulation of nucleotide metabolic process

skeletal morphogenesis

antigen processing and presentation, exogenous lipid ar

T-helper 2 type immune response

embryonic gut development

glucosamine metabolic process

ectodermal gut development

digestive tract morphogenesis

rRNA processing

menstrual cycle phase

estrogen metabolic process

oocyte differentiation

negative regulation of protein transport

somatic cell DNA recombination

sphingolipid metabolic process

indole derivative biosynthetic process

establishment of nucleus localization

forelimb morphogenesis

phospholipid dephosphorylation

\begin{tabular}{|c|c|c|}
\hline 0 & 1 & 1 \\
\hline 0 & 1 & 1 \\
\hline 0.62 & 1 & 1 \\
\hline 0.31 & 1 & 1 \\
\hline 0 & 1 & 1 \\
\hline 0 & 1 & 1 \\
\hline 0.31 & 1 & 1 \\
\hline 0 & 1 & 1 \\
\hline 0 & 1 & 1 \\
\hline 0 & 1 & 1 \\
\hline 0.62 & 1 & 1 \\
\hline 0 & 1 & 1 \\
\hline 0 & 1 & 1 \\
\hline 0 & 1 & 1 \\
\hline 0.31 & 1 & 1 \\
\hline 0 & 1 & 1 \\
\hline 0 & 1 & 1 \\
\hline 0 & 1 & 1 \\
\hline 0.31 & 1 & 1 \\
\hline 0 & 1 & 1 \\
\hline 0 & 1 & 1 \\
\hline 0.31 & 1 & 1 \\
\hline 0.31 & 1 & 1 \\
\hline 0.31 & 1 & 1 \\
\hline 0.31 & 1 & 1 \\
\hline 0 & 1 & 1 \\
\hline 0 & 1 & 1 \\
\hline 0 & 1 & 1 \\
\hline 0.62 & 1 & 1 \\
\hline 0 & 1 & 1 \\
\hline 0 & 1 & 1 \\
\hline 0 & 1 & 1 \\
\hline 0 & 1 & 1 \\
\hline 0 & 1 & 1 \\
\hline 0 & 1 & 1 \\
\hline 0 & 1 & 1 \\
\hline
\end{tabular}

lipid glycosylation

acetyl-CoA catabolic process 
ATP-dependent proteolysis

sterol metabolic process

positive regulation of protein transport

cholesterol transport

establishment of vesicle localization

germ-line stem cell division

endosome transport

glycolate metabolic process

telomere organization and biogenesis

dorsal/ventral axis specification

amino acid transport

regulation of lipid kinase activity

gonadotropin secretion

purine nucleotide biosynthetic process

anaphase

\section{rRNA modification}

regulation of synapse structure and activity

negative regulation of protein secretion

purine base biosynthetic process

peptidyl-amino acid modification

positive regulation of inflammatory response

coenzyme A metabolic process

oogenesis (sensu Mammalia)

interleukin-1 secretion

protein kinase B signaling cascade

nucleoside phosphate metabolic process

frizzled-2 signaling pathway

protein maturation via proteolysis

nucleoside diphosphate biosynthetic process

sister chromatid segregation

disruption by symbiont of host cells

gamma-delta $T$ cell activation

catecholamine metabolic process

\begin{tabular}{|c|c|}
\hline 0 & 1 \\
\hline 0.31 & 1 \\
\hline 0.31 & 1 \\
\hline 0 & 1 \\
\hline 0.62 & 1 \\
\hline 0 & 1 \\
\hline 0 & 1 \\
\hline 0.31 & 1 \\
\hline 0 & 1 \\
\hline 0.31 & 1 \\
\hline 0 & 1 \\
\hline 0 & 1 \\
\hline 0 & 1 \\
\hline 0.31 & 1 \\
\hline 0 & 1 \\
\hline 0 & 1 \\
\hline 0.62 & 1 \\
\hline 0 & 1 \\
\hline 0 & 1 \\
\hline 0 & 1 \\
\hline 0 & 1 \\
\hline 0 & 1 \\
\hline 1.55 & 1 \\
\hline 0 & 1 \\
\hline 0 & 1 \\
\hline 0 & 1 \\
\hline 0 & 1 \\
\hline 0 & 1 \\
\hline 0.31 & 1 \\
\hline 0 & 1 \\
\hline 0 & 1 \\
\hline 0 & 1 \\
\hline 0.31 & 1 \\
\hline 0 & 1 \\
\hline 0 & 1 \\
\hline 0.62 & 1 \\
\hline
\end{tabular}


RNA 3'-end processing

CAMP metabolic process

isoprenoid biosynthetic process

regulation of cytokine and chemokine mediated signalin

indolalkylamine metabolic process

positive regulation of GTPase activity

positive regulation of response to tumor cell

polarized epithelial cell differentiation

di-, tri-valent inorganic anion homeostasis

$\mathrm{M}$ phase of mitotic cell cycle

spermatid differentiation

protein modification by small protein conjugation

re-entry into mitotic cell cycle

regulation of lymphocyte proliferation

thiamin diphosphate metabolic process

thiamin and derivative biosynthetic process

RNA transport

deoxyribonucleoside catabolic process

development of primary male sexual characteristics

somatic diversification of immune receptors

neural plate morphogenesis

tricarboxylic acid cycle intermediate metabolic process

purine transport

chondroitin sulfate proteoglycan metabolic process

metaphase

organic cation transport

neural crest cell development

regulation of blood coagulation

regulation of smooth muscle cell differentiation

oogenesis stage

purine ribonucleotide metabolic process

\begin{tabular}{|c|c|c|}
\hline 0 & 1 & 1 \\
\hline 2.17 & 1 & 1 \\
\hline 0 & 1 & 1 \\
\hline 0 & 1 & 1 \\
\hline 0 & 1 & 1 \\
\hline 0 & 1 & 1 \\
\hline 0.31 & 1 & 1 \\
\hline 0 & 1 & 1 \\
\hline 0 & 1 & 1 \\
\hline 0 & 1 & 1 \\
\hline 0 & 1 & 1 \\
\hline 0 & 1 & 1 \\
\hline 0 & 1 & 1 \\
\hline 0 & 1 & 1 \\
\hline 1.55 & 1 & 1 \\
\hline 0 & 1 & 1 \\
\hline 0.62 & 1 & 1 \\
\hline 0 & 1 & 1 \\
\hline 0.62 & 1 & 1 \\
\hline 0 & 1 & 1 \\
\hline 0 & 1 & 1 \\
\hline 0.31 & 1 & 1 \\
\hline 0 & 1 & 1 \\
\hline 0.31 & 1 & 1 \\
\hline 0.31 & 1 & 1 \\
\hline 0.31 & 1 & 1 \\
\hline 0.31 & 1 & 1 \\
\hline 0 & 1 & 1 \\
\hline 0 & 1 & 1 \\
\hline 0 & 1 & 1 \\
\hline 0 & 1 & 1 \\
\hline 0 & 1 & 1 \\
\hline 0 & 1 & 1 \\
\hline 0 & 1 & 1 \\
\hline 0 & 1 & 1 \\
\hline 0.62 & 1 & 1 \\
\hline
\end{tabular}


butyrate metabolic process

lymphocyte mediated immunity

PML body organization and biogenesis

positive regulation of lymphocyte activation

regulation of hormone secretion

positive regulation of vasodilation

protein amino acid sulfation

uroporphyrinogen III biosynthetic process

protein stabilization

platelet activation

negative regulation of muscle cell differentiation

regulation of $B$ cell activation

tissue morphogenesis

positive regulation of ion transport

lung development

histidine family amino acid metabolic process

protein heterooligomerization

establishment of cell polarity

midgut development

pyrimidine nucleotide-sugar transport

membrane protein ectodomain proteolysis

modification of cellular component in other organism dur

pyrimidine base catabolic process

sister chromatid cohesion

aromatic amino acid family metabolic process

RNA editing

benzoate metabolic process

phagocytosis

specification of organ identity

T cell proliferation

modification by symbiont of host structure

vitamin B6 biosynthetic process

formate metabolic process

tricarboxylic acid transport

branched chain family amino acid metabolic process

conditioned taste aversion

\begin{tabular}{|c|c|c|}
\hline 0 & 1 & 1 \\
\hline 0.62 & 1 & 1 \\
\hline 0 & 1 & 1 \\
\hline 0.62 & 1 & 1 \\
\hline 0 & 1 & 1 \\
\hline 0 & 1 & 1 \\
\hline 0 & 1 & 1 \\
\hline 0 & 1 & 1 \\
\hline 0 & 1 & 1 \\
\hline 0 & 1 & 1 \\
\hline 0 & 1 & 1 \\
\hline 0.31 & 1 & 1 \\
\hline 0.62 & 1 & 1 \\
\hline 0 & 1 & 1 \\
\hline 0.62 & 1 & 1 \\
\hline 0.31 & 1 & 1 \\
\hline 0 & 1 & 1 \\
\hline 0 & 1 & 1 \\
\hline 0 & 1 & 1 \\
\hline 0 & 1 & 1 \\
\hline 0 & 1 & 1 \\
\hline 0 & 1 & 1 \\
\hline 0 & 1 & 1 \\
\hline 0 & 1 & 1 \\
\hline 0.31 & 1 & 1 \\
\hline 0 & 1 & 1 \\
\hline 0 & 1 & 1 \\
\hline 0.31 & 1 & 1 \\
\hline 0.31 & 1 & 1 \\
\hline 0.93 & 1 & 1 \\
\hline 0 & 1 & 1 \\
\hline 0 & 1 & 1 \\
\hline 0 & 1 & 1 \\
\hline 0 & 1 & 1 \\
\hline 0 & 1 & 1 \\
\hline 0 & 1 & 1 \\
\hline
\end{tabular}


beta-amyloid metabolic process

immunoglobulin production

purine ribonucleoside metabolic process

pyrimidine deoxyribonucleoside metabolic process

segment specification

regulation of phosphate metabolic process

monocarboxylic acid transport

establishment and/or maintenance of apical/basal cell pc

quinone cofactor biosynthetic process

regulation of immune response to tumor cell 
Gene Ontology : biological process. Level: 8 ossification

proteolysis involved in cellular protein catabolic process activation of phospholipase A2

double-strand break repair via homologous recombinatic heparan sulfate proteoglycan biosynthetic process

DNA strand elongation during DNA replication

\section{protein targeting}

positive regulation of transcription

skeletal muscle development

regulation of programmed cell death

cell cycle arrest

BMP signaling pathway

detection of light stimulus during visual perception

activated $\mathrm{T}$ cell proliferation

catecholamine biosynthetic process

diacylglycerol metabolic process

glycolipid catabolic process

positive regulation of small GTPase mediated signal trar

cGMP biosynthetic process

epidermis development

regulation of cyclin-dependent protein kinase activity

regulation of synaptic plasticity

glial cell differentiation

intracellular protein transport across a membrane

sodium ion transport

inner ear development

\section{apoptosis}

neuron differentiation

phosphate transport

protein amino acid glycosylation

regulation of cell growth

positive regulation of Notch signaling pathway

asparagine metabolic process

regulation of DNA ligation

phagocytosis, recognition

Percentage of genes with allele-specific expression

97

2.36

0

0

0

2.92

0.83

7.78

1.39

0.14

0.14

0.14

0.14

0.14

0.14

0.14

2.22

0.42

0.42

1.53

0.56

10.56

6.81

1.25

2.92

2.08
Percentage of genes without allele-specific expression

.45

3.45

4.98
0.77

0.77
0.77

0.77

0.77

5.36

2.68

2.3

4.98

2.68

0.77

0.77

0.77

0.77

0.77

0.77

0.77

0.77

0.77

1.15

1.15

1.15

1.15

0.38

1.53

7.66

4.6

2.3

2.3
1.53

0.77

0.38

0.38

0.38

0.38 nadjusted pvalue Adjusted pvalueFDR

0.01768197

0.0559508

0.07058603

0.07058603

0.07058603

0.07058603

0.07934332

0.08691233

0.09400779

0.1574026

0.1738405

0.1744102

0.1744102

0.1744102

0.1744102

0.1744102

0.1744102

0.1744102

0.1744102

0.1800566

0.195049

0.195049

0.195049

0.195049

0.1993123

0.219653

0.2235061

0.2334345

0.2451004

0.2607733

0.2657904

0.266055

0.266055

0.266055

0.266055
1

1

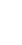

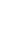

1

1

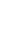

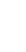

1

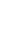

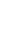

1

1

1 
vasoconstriction of artery during baroreceptor response

inositol phosphate-mediated signaling

low-density lipoprotein catabolic process

bile acid biosynthetic process

mesoderm morphogenesis

fatty acid desaturation

actomyosin structure organization and biogenesis

bile acid catabolic process

transcription from RNA polymerase I promoter

regulation of rhodopsin gene activity

catecholamine catabolic process

platelet activating factor metabolic process

DNA replication initiation

aspartate family amino acid biosynthetic process

phospholipid catabolic process

phospholipid transfer to membrane

antibacterial humoral response (sensu Vertebrata)

centrosome separation

protein deglycosylation

glucan catabolic process

ventricular system development

increased strength of heart contraction by epinephrine-n

inductive cell-cell signaling

double-strand break repair via nonhomologous end joini

DNA unwinding during replication

positive regulation of vesicle fusion

periodic partitioning

positive regulation of urothelial cell proliferation

transcription from mitochondrial promoter

antifungal humoral response (sensu Vertebrata)

positive regulation of heart contraction rate by epinephri

DNA damage induced protein phosphorylation

striated muscle cell differentiation

protein deubiquitination

galactolipid metabolic process

sterol catabolic process 
extracellular polysaccharide biosynthetic process

lipopolysaccharide biosynthetic process

negative regulation of macrophage activation

transcription from RNA polymerase II promoter

sphingolipid catabolic process

glycosaminoglycan biosynthetic process

negative regulation of $\mathrm{T}$ cell activation

chloride transport

regulation of transcription factor activity

glycerophospholipid metabolic process

nuclear export

glutamine metabolic process

glycogen metabolic process

female gonad development

post-Golgi vesicle-mediated transport

eye development (sensu Vertebrata)

hexose catabolic process

chromatin assembly or disassembly

G-protein signaling, coupled to IP3 second messenger (

interleukin-6 biosynthetic process

ransforming growth factor beta receptor signaling path

transcription initiation

positive regulation of protein kinase activity

actin filament organization

calcium ion transport

protein autoprocessing

icosanoid metabolic process

ear morphogenesis

negative regulation of protein kinase activity

protein amino acid lipidation

activation of immune response

glutamine family amino acid biosynthetic process

male gonad development

$S$ phase of mitotic cell cycle

negative regulation of progression through mitotic cell c)

mast cell mediated immunity

\begin{tabular}{ll}
0.266055 & 1 \\
0.266055 & 1 \\
0.266055 & 1 \\
0.2689963 & 1 \\
0.2889035 & 1 \\
0.2889035 & 1 \\
0.2889035 & 1 \\
0.2889035 & 1 \\
0.2889035 & 1 \\
0.2889035 & 1 \\
0.2889035 & 1 \\
0.2889035 & 1 \\
0.2889035 & 1 \\
0.2889035 & 1 \\
0.2889035 & 1 \\
0.2889035 & 1 \\
0.3055197 & 1 \\
0.3204053 & 1 \\
0.3204053 & 1 \\
0.3328018 & 1 \\
0.3328018 & 1 \\
0.3328018 & 1 \\
0.3491708 & 1 \\
0.3504891 & 1 \\
0.3576195 & 1 \\
0.3909792 & 1 \\
0.3909792 & 1 \\
0.3909792 & 1 \\
0.4449706 & 1 \\
0.4449706 & 1 \\
0.4582135 & 1 \\
0.4615241 & 1 \\
0.4615241 & 1 \\
0.4615241 & 1 \\
0.4615241 & 1 \\
0.4615241 & 1 \\
0 & 1 \\
& 1 \\
\hline
\end{tabular}


CAMP biosynthetic process

ARF protein signal transduction

intra-Golgi vesicle-mediated transport

insulin-like growth factor receptor signaling pathway

androgen biosynthetic process

Mo-molybdopterin cofactor biosynthetic process

regulation of JAK-STAT cascade

sulfur amino acid biosynthetic process

synaptic transmission, dopaminergic

$T$ cell activation during immune response

Rac protein signal transduction

regulation of RNA stability

natural killer cell mediated immunity

aromatic amino acid family catabolic process

RNA splicing, via endonucleolytic cleavage and ligation

transcription from RNA polymerase III promoter

acidic amino acid transport

triacylglycerol metabolic process

L-phenylalanine metabolic process

acylglycerol biosynthetic process

G-protein signaling, coupled to cyclic nucleotide second

chromatin modification

ureteric bud development

negative regulation of transcription

peptidyl-tyrosine modification

potassium ion transport

RNA aminoacylation

serine family amino acid biosynthetic proces

ibonucleoside monophosphate biosynthetic process

gastrulation (sensu Vertebrata) 
cortical actin cytoskeleton organization and biogenesis positive regulation of cytokine secretion

regulation of positive chemotaxis

negative regulation of $B$ cell activation

hexose biosynthetic process

maintenance of fidelity during DNA-dependent DNA repl

folic acid and derivative biosynthetic process

embryonic digit morphogenesis

positive regulation of chemotaxis

actin filament-based movement

pyridine nucleotide biosynthetic process

ER to Golgi vesicle-mediated transport

DNA integrity checkpoint

fucose metabolic process

transition metal ion homeostasis

nicotinamide metabolic process

regulation of striated muscle development

spindle organization and biogenesis

cAMP-mediated signaling

nuclear import

calcium ion homeostasis

sphingoid metabolic process

positive regulation of phosphate metabolic process

interleukin-2 biosynthetic process

axon ensheathment

insulin receptor signaling pathway

cell projection biogenesis

protein ubiquitination

positive regulation of lymphocyte proliferation

androgen receptor signaling pathway

regulation of neurogenesis

regulation of MAPK activity

hemopoiesis

positive regulation of protein biosynthetic process

regulation of cytokine biosynthetic process

glucose metabolic process

\begin{tabular}{|c|c|}
\hline 0.5693293 & \\
\hline 0.5693293 & \\
\hline 0.5693293 & \\
\hline 0.5693293 & \\
\hline 0.5693293 & \\
\hline 0.5693293 & \\
\hline 0.5693293 & \\
\hline 0.5693293 & \\
\hline 0.5693293 & \\
\hline 0.5693293 & \\
\hline 0.5693293 & \\
\hline 0.57843 & \\
\hline 0.57843 & \\
\hline 0.57843 & \\
\hline 0.57843 & \\
\hline 0.57843 & \\
\hline 0.57843 & \\
\hline 0.57843 & \\
\hline 0.5801394 & \\
\hline 0.5901221 & \\
\hline 0.6053717 & \\
\hline 0.6132732 & \\
\hline 0.6132732 & \\
\hline 0.6132732 & \\
\hline 0.6132732 & \\
\hline 0.6595536 & \\
\hline 0.6595536 & \\
\hline 0.6595536 & \\
\hline 0.6595536 & \\
\hline 0.68225 & \\
\hline 0.68225 & \\
\hline 0.7065722 & \\
\hline 0.7110969 & \\
\hline 0.7309491 & \\
\hline 0.7543467 & \\
\hline 0.7705619 & \\
\hline
\end{tabular}


regulation of I-kappaB kinase/NF-kappaB cascade microtubule-based movement

regulation of Ras protein signal transduction

Rho protein signal transduction

blood vessel morphogenesis

regulation of transcription, DNA-dependent

chondroitin sulfate metabolic process

eye development (sensu Endopterygota)

lymphocyte costimulation

positive regulation of $B$ cell activation

interleukin-1 biosynthetic process

regulation of DNA recombination

acyl-CoA metabolic process

neurotransmitter metabolic process

negative regulation of smoothened signaling pathway

somatic diversification of immunoglobulins

neurite regeneration

lysosomal transport

ammonium transport

protein depalmitoylation

purine ribonucleoside diphosphate metabolic process

negative regulation of protein biosynthetic process

synaptic transmission, glutamatergic

cysteine metabolic process

'de novo' pyrimidine base biosynthetic process

cholesterol metabolic process

estrogen catabolic process

positive regulation of epithelial cell differentiation

purine nucleoside diphosphate biosynthetic process

regulation of leukocyte degranulation

positive regulation of $\mathrm{T}$ cell activation

regulation of DNA replication

receptor internalization

meiosis

mitosis

chondroitin sulfate proteoglycan biosynthetic process

\begin{tabular}{|c|c|}
\hline 1.15 & 0.7705619 \\
\hline 1.53 & 0.7706805 \\
\hline 1.92 & 0.8102265 \\
\hline 1.92 & 0.8115185 \\
\hline 3.07 & 0.8435508 \\
\hline 27.59 & 0.8709582 \\
\hline 0 & 1 \\
\hline 0 & 1 \\
\hline 0 & 1 \\
\hline 0.38 & 1 \\
\hline 0 & 1 \\
\hline 0 & 1 \\
\hline 0 & 1 \\
\hline 0.77 & 1 \\
\hline 0 & 1 \\
\hline 0.38 & 1 \\
\hline 0 & 1 \\
\hline 0.38 & 1 \\
\hline 0 & 1 \\
\hline 0 & 1 \\
\hline 0 & 1 \\
\hline 0.38 & 1 \\
\hline 0 & 1 \\
\hline 0 & 1 \\
\hline 0 & 1 \\
\hline 0.77 & 1 \\
\hline 0 & 1 \\
\hline 0 & 1 \\
\hline 0 & 1 \\
\hline 0 & 1 \\
\hline 0.77 & 1 \\
\hline 0 & 1 \\
\hline 0 & 1 \\
\hline 0.77 & 1 \\
\hline 1.92 & 1 \\
\hline 0 & 1 \\
\hline
\end{tabular}


sperm chromatin decondensation

RNA interference

interleukin-8 biosynthetic process

embryonic forelimb morphogenesis

peroxisomal long-chain fatty acid import

pyrimidine deoxyribonucleoside catabolic process

fatty acid biosynthetic process

regulation of lymphocyte mediated immunity

positive regulation of protein modification

male genitalia developmen

regulation of cyclic nucleotide metabolic process

glial cell migration

epidermal growth factor receptor signaling pathway

mRNA transcription

embryonic ectodermal gut development

estrogen biosynthetic process

sodium ion homeostasis

macrophage chemotaxis

protein amino acid methylation

induction of an organ

regulation of cell killing

UDP-N-acetylglucosamine transport

killing of cells of another organism

purine ribonucleotide biosynthetic process

patched ligand processing

regulation of fatty acid metabolic process

negative regulation of lymphocyte proliferation

neuroblast proliferation

midbrain development

arginine metabolic process

interleukin-13 biosynthetic process

neuron migration

fatty acid oxidation

modification by symbiont of host cellular component

establishment of apical/basal cell polarity

beta-tubulin folding 
transcription-coupled nucleotide-excision repair

peptidyl-threonine modification

UDP-xylose transport

chondrocyte differentiation

succinyl-CoA metabolic process

amyloid precursor protein metabolic process

histidine family amino acid catabolic process

tripartite regional subdivision

oocyte development

regulation of gamma-delta $T$ cell activation

pyrimidine ribonucleotide biosynthetic process

interleukin-12 biosynthetic process

$\mathrm{N}$-acetylglucosamine metabolic process

ribonucleoside diphosphate biosynthetic process

phospholipid biosynthetic process

L-amino acid transport

negative regulation of smooth muscle cell differentiation

tricarboxylic acid cycle

negative regulation of nucleotide metabolic process

regulation of $B$ cell proliferation

negative regulation of amino acid metabolic process

negative regulation of hormone secretion

neural crest cell migration

establishment of lymphocyte polarity

interleukin-1 beta secretion

ribonucleoside triphosphate biosynthetic process

regulation of translational initiation

mRNA transport

protein sumoylation

mitochondrial DNA replication

\begin{tabular}{|c|c|}
\hline 0 & 1 \\
\hline 0 & 1 \\
\hline 0.38 & 1 \\
\hline 0 & 1 \\
\hline 0.77 & 1 \\
\hline 0 & 1 \\
\hline 0 & 1 \\
\hline 0 & 1 \\
\hline 0 & 1 \\
\hline 0 & 1 \\
\hline 0.38 & 1 \\
\hline 0 & 1 \\
\hline 0.38 & 1 \\
\hline 0 & 1 \\
\hline 0 & 1 \\
\hline 0 & 1 \\
\hline 0.38 & 1 \\
\hline 0.38 & 1 \\
\hline 0 & 1 \\
\hline 0 & 1 \\
\hline 0.77 & 1 \\
\hline 0 & 1 \\
\hline 0 & 1 \\
\hline 0 & 1 \\
\hline 0 & 1 \\
\hline 0.38 & 1 \\
\hline 0 & 1 \\
\hline 0 & 1 \\
\hline 0 & 1 \\
\hline 0 & 1 \\
\hline 0 & 1 \\
\hline 0.77 & 1 \\
\hline 0.38 & 1 \\
\hline 0.38 & 1 \\
\hline 0 & 1 \\
\hline 0 & 1 \\
\hline
\end{tabular}


positive regulation of progression through mitotic cell cyc

embryonic digestive tract morphogenesis

purine ribonucleoside triphosphate metabolic process

long-chain fatty acid metabolic process

gut morphogenesis

uracil catabolic process

protein amino acid acetylation

chaperonin-mediated tubulin folding

positive regulation of DNA metabolic process

regulation of phosphorylation

bile acid transport

chemokine biosynthetic process

negative regulation of phosphate metabolic process

endoderm formation

vesicle targeting

negative regulation of small GTPase mediated signal tra

pyrimidine ribonucleoside triphosphate metabolic proces

peptidyl-asparagine modification

hyaluronan metabolic process

tyrosine metabolic process

neutral amino acid transport

microtubule bundle formation

signal peptide processing

somatic diversification of immune receptors via germline

nerve growth factor receptor signaling pathway

leukocyte mediated cytotoxicity

retrograde transport, endosome to Golgi

regulation of mitochondrial membrane permeability

odontogenesis (sensu Vertebrata)

transition metal ion transport

regulation of phosphoinositide 3-kinase activity

cartilage condensation

regulation of alpha-beta $\mathrm{T}$ cell activation

\begin{tabular}{|c|c|}
\hline 0 & 1 \\
\hline 0 & 1 \\
\hline 0 & 1 \\
\hline 0 & 1 \\
\hline 0.38 & 1 \\
\hline 0.77 & 1 \\
\hline 0 & 1 \\
\hline 0.38 & 1 \\
\hline 0 & 1 \\
\hline 0 & 1 \\
\hline 0 & 1 \\
\hline 0.38 & 1 \\
\hline 0.77 & 1 \\
\hline 0 & 1 \\
\hline 0 & 1 \\
\hline 0 & 1 \\
\hline 0 & 1 \\
\hline 0.38 & 1 \\
\hline 0 & 1 \\
\hline 0.38 & 1 \\
\hline 0.38 & 1 \\
\hline 0 & 1 \\
\hline 0 & 1 \\
\hline 0 & 1 \\
\hline 0 & 1 \\
\hline 0 & 1 \\
\hline 0 & 1 \\
\hline 0 & 1 \\
\hline 0.38 & 1 \\
\hline 0 & 1 \\
\hline 0 & 1 \\
\hline 0.38 & 1 \\
\hline 0.38 & 1 \\
\hline 0 & 1 \\
\hline 0 & 1 \\
\hline 0 & 1 \\
\hline
\end{tabular}


fibroblast growth factor receptor signaling pathway

signal complex formation

phosphate ion homeostasis

tumor necrosis factor-beta biosynthetic process $\quad 0.28$

peptidyl-histidine modification $\quad 0.14$

primary follicle stage, oogenesis (sensu Mammalia)

$\begin{array}{ll}\text { regulation of nucleotide biosynthetic process } & 0.14\end{array}$

regulation of MAPKKK cascade $\quad 0.28$

neurotransmitter secretion $\quad 1.53$

positive regulation of exocytosis $\quad 0.28$

estrogen receptor signaling pathway $\quad 0.14$

regulation of MHC class II biosynthetic process

RNA polyadenylation 0.28

positive regulation of immune response to tumor cell

regulation of pinocytosis $\quad 0.28$

actin cytoskeleton reorganization $\quad 0.14$

Notch receptor processing 0.14

ephrin receptor signaling pathway $\quad 0.14$

mannose metabolic process $\quad 0.28$

$\begin{array}{ll}\text { malate metabolic process } & 0.28\end{array}$

positive regulation of leukocyte mediated immunity $\quad 0.14$

$\begin{array}{ll}\text { lymph node development } & 0.14\end{array}$

rostrocaudal neural tube patterning 0.14

negative regulation of blood coagulation 0.28

CGMP catabolic process $\quad 0.14$

lymphocyte chemotaxis 0.28

\begin{tabular}{ll} 
L-serine metabolic process & 0.28 \\
\hline
\end{tabular}

succinate transport $\quad 0.14$

regulation of gonadotropin secretion $\quad 0.14$

actin polymerization and/or depolymerization 1.11

alpha-beta $\mathrm{T}$ cell proliferation 
branched chain family amino acid catabolic process

base conversion or substitution editing

base-excision repair, gap-filling

G2 phase of mitotic cell cycle

eye morphogenesis

proton transport

regulation of $\mathrm{T}$ cell proliferation

posterior midgut development

glucose transport

B cell mediated immunity

intermediate mesoderm development

negative regulation of cytokine and chemokine mediatec

thiamin diphosphate biosynthetic process

glucan biosynthetic process

citrate transport

RNA splicing, via transesterification reactions

regulation of catecholamine metabolic process

JNK cascade

regulation of calcium-mediated signaling

glycosphingolipid metabolic process

fructose metabolic process

follicle-stimulating hormone secretion

mRNA editing

histidine metabolic process

embryonic skeletal morphogenesis

G2/M transition of mitotic cell cycle

negative regulation of Wnt receptor signaling pathway

indolalkylamine biosynthetic process

establishment and/or maintenance of epithelial cell polar 
pyridoxine biosynthetic process

granulocyte macrophage colony-stimulating factor biosyl

forebrain development

purine ribonucleoside salvage

ubiquinone biosynthetic process
positive regulation of hormone secretion

$T$ cell mediated immunity

vascular endothelial growth factor receptor signaling pat

regulation of actin filament length

negative regulation of DNA metabolic process

entry into host cell

opsonization

coenzyme A biosynthetic process

glycolipid biosynthetic process

protein heterotetramerization

sphingolipid biosynthetic process

arm morphogenesis

mRNA 3'-end processing

regulation of nucleocytoplasmic transport

G1/S transition of mitotic cell cycle

fused antrum stage, oogenesis (sensu Mammalia)

I-kappaB phosphorylation

neural tube formation

eosinophil chemotaxis

regulation of interleukin-1 secretion

male germ-line stem cell division

neutrophil chemotaxis

C21-steroid hormone biosynthetic process

tryptophan metabolic process

interleukin-3 biosynthetic process

cytoplasmic sequestering of protein

positive regulation of innate immune response 
Gene Ontology : biological process. Level: 9 regulation of ossification

bone mineralization

positive regulation of transcription, DNA-dependent

Percentage of genes with allele-specific expressio

positive regulation of programmed cell death

phototransduction

calcium-dependent phospholipase A2 activation

blood vessel remodeling

chromatin assembly

collagen catabolic process

regulation of apoptosis

regulation of transcription from RNA polymerase II pron

modification-dependent protein catabolic process

skeletal muscle fiber development

neuron fate commitment

regulation of neuronal synaptic plasticity

protein amino acid O-linked glycosylation

synaptic vesicle exocytosis

protein export from nucleus

phosphoinositide metabolic process

hair follicle development

regulation of mitosis

triacylglycerol biosynthetic process

striated muscle cell developmen

egulation of transcription from RNA polymerase I prom

S-adenosylmethionine biosynthetic process

neurotransmitter catabolic process

mesoderm formation

sphingoid catabolic process

Schwann cell differentiation

positive regulation of $B$ cell proliferation

protein targeting to peroxisome

elencephalon development

positive regulation of DNA ligation

endochondral ossification

protein autoubiquitination
Percentage of genes without allele-specific expression

Unadjusted pvalue Adjusted pvalueFDR

8.87

6.9

0

0.49

13.55

14.29

3.69

1.48

0.25

0.25

0.25

0.25

0.25

1.72

1.48

0

0

0

0

0

0

0

0

0 $\begin{array}{lll}2 & 0.01934914 & 1\end{array}$

$2 \quad 0.01934914$

$3.33 \quad 0.02764696$

$2.67 \quad 0.0648839$

$1.33-0.07242854$

$1.33 \quad 0.07242854$

$2.67 \quad 0.08893205$

$2 \quad 0.1245022$

$8.67-0.1445579$

$9.33 \quad 0.1541752$

$6.67 \quad 0.1647821$

$3.33 \quad 0.1770124$

$1.33 \quad 0.1785873$

$1.33 \quad 0.1785873$

$1.33 \quad 0.1785873$

$1.33-0.1785873$

$1.33 \quad 0.1785873$

$1.33 \quad 0.1785873$

$0 \quad 0.1980866$

$0 \quad 0.1981695$

$0.67 \quad 0.2697842$

$0.67 \quad 0.2697842$

$0.67 \quad 0.2697842$

$0.67 \quad 0.2697842$

$0.67 \quad 0.2697842$

$0.67 \quad 0.2697842$

$0.67 \quad 0.2697842$

$0.67 \quad 0.2697842$

$0.67 \quad 0.2697842$

$0.67 \quad 0.2697842$

$0.67 \quad 0.2697842$

$0.67 \quad 0.2697842$
1

1

1

1

1

1

1

1

1

1

1

1

1

1

1
$1.33 \quad 0.07242854$

$0.67 \quad 0.2697842$ 
retrograde transport, vesicle recycling within Golgi

platelet activating factor biosynthetic process

positive regulation of gliogenesis

cholesterol catabolic process

ovulation (sensu Mammalia)

asparagine biosynthetic process

oxidized low-density lipoprotein catabolic process

segment polarity determination

mitotic centrosome separation

negative regulation of transcription factor activity

galactolipid catabolic process

glycogen catabolic process

fatty acid beta-oxidation

glutamine biosynthetic process

regulation of CGMP metabolic process

lagging strand elongation

Golgi to plasma membrane transport

eukotriene metabolic process

protein prenylation

eye development (sensu Mammalia)

ureteric bud branching

neurotransmitter biosynthetic process

actin filament depolymerization

positive regulation of neurogenesis

activation of protein kinase activity

G-protein signaling, coupled to CAMP nucleotide secon

inner ear morphogenesis

protein amino acid autophosphorylation

neuron development

negative regulation of programmed cell death

cytosolic calcium ion homeostasis

regulation of activated $\mathrm{T}$ cell proliferation

astrocyte differentiation

-phenylalanine catabolic process

mast cell degranulation

norepinephrine metabolic process

0.2697842

0.2697842

0.2952085

0.2952085

0.2952085

0.2952085

0.2952085

0.2952085

0.3307322

0.3510684

0.3755438

0.3940697

0.3940697

0.3955965

0.4111837

0.4503502

0.4671398

0.4671398

0.4671398

0.4671398

0.4671398 
substrate-bound cell migration, cell extension $\quad 0.25$

positive regulation of JAK-STAT cascade $\quad 0.25$

glutamate transport

regulation of glial cell differentiation

apocarotenoid metabolic process

negative regulation of neurogenesis

positive regulation of receptor mediated endocytosis

negative regulation of cyclin-dependent protein kinase :

tRNA splicing

regulation of interferon-gamma biosynthetic process

regulation of BMP signaling pathway

regulation of calcium ion transport

regulation of mRNA stability

diacylglycerol biosynthetic process

glycosphingolipid catabolic process

axon cargo transport

angiogenesis

peptidyl-tyrosine phosphorylation

L-fucose metabolic process

positive regulation of positive chemotaxis

mismatch repair

tRNA aminoacylation for protein translation

energy coupled proton transport, down electrochemical

actin filament bundle formation

mmune response-activating signal transduction

DNA damage checkpoint

ranscription initiation from RNA polymerase II promote

negative regulation of cell growth

regulation of interleukin- 6 biosynthetic process

chromatin remodeling

protein import into nucleus

meiosis I

$\begin{array}{rr}0.67 & 0.4671398 \\ 0.67 & 0.4671398 \\ 0.67 & 0.4671398 \\ 0.67 & 0.4671398 \\ 0.67 & 0.4671398 \\ 0.67 & 0.4671398 \\ 0.67 & 0.4671398 \\ 0.67 & 0.4671398 \\ 0.67 & 0.4671398 \\ 0.67 & 0.4671398 \\ 0.67 & 0.4671398 \\ 0.67 & 0.4671398 \\ 0.67 & 0.4671398 \\ 0.67 & 0.4671398 \\ 0.67 & 0.4671398 \\ 0.67 & 0.4671398 \\ 0.67 & 0.4671398 \\ 0.67 & 0.4671398 \\ 0.67 & 0.4671398 \\ 0.67 & 0.4671398 \\ 3.33 & 0.4971736 \\ 2.67 & 0.4983115 \\ 0 & 0.5671713 \\ 0 & 0.5671713 \\ 0 & 0.5671713 \\ 0 & 0.5671713 \\ 0 & 0.5671713 \\ 0 & 0.5671713 \\ 0 & 0.5783899 \\ 0 & 0.5783899 \\ 0 & 0.5783899 \\ 0 & 0.5783899 \\ 0 & 0.5783899 \\ 0 & 0.5783899 \\ 4 & 0.5901606 \\ 1.33 & 0.6152426 \\ & \end{array}$


myelination

dopamine metabolic process

actin filament polymerization

positive regulation of $\mathrm{T}$ cell proliferation

regulation of actin polymerization and/or depolymerizati

epidermis morphogenesis

protein amino acid N-linked glycosylation

glucose catabolic process

positive regulation of cytokine biosynthetic process

positive regulation of I-kappaB kinase/NF-kappaB casc

regulation of Rho protein signal transduction

leukocyte differentiation

negative regulation of transcription, DNA-dependent

neutrophil apoptosis

regulation of granulocyte macrophage colony-stimulatir

regulation of microtubule polymerization or depolymeriz

positive regulation of translational initiation

ectodermal gut morphogenesis

peptidyl-tyrosine sulfation

RNA export from nucleus

endoplasmic reticulum calcium ion homeostasis

primary neural tube formation

Cdc42 protein signal transduction

catecholamine uptake during transmission of nerve imp

NADP metabolic process

amyloid precursor protein catabolic process

entry of virus into host cell

copper ion homeostasis

regulation of interleukin- 13 biosynthetic process

glucose import

GTP metabolic process

thymidine catabolic process

0.6889394

0.6889394

0.6889394

0.7080766

0.7684468

0.7910422

0.8155387

0.8485424 
transcription initiation from RNA polymerase III promot€

energy coupled proton transport, against electrochemic

regulation of transforming growth factor beta receptor $\mathrm{s}$

regulation of JNK activity

glutamate secretion

mitotic spindle organization and biogenesis

skin development

vasculogenesis

misfolded or incompletely synthesized protein catabolic

regulation of leukocyte mediated cytotoxicity

$\begin{array}{ll}\text { L-serine biosynthetic process } & 0.49\end{array}$

regulation of protein amino acid phosphorylation $\quad 1.23$

gastrulation (sensu Mammalia) $\quad 0.49$

microtubule polymerization

spermatid nuclear differentiation

cytolysis of cells of another organism

lamellipodium biogenesis

mitotic anaphase

positive regulation of lymphocyte mediated immunity

killing of cells in other organism during symbiotic interar

positive regulation of pinocytosis

microtubule depolymerization

fucose biosynthetic process

natural killer cell mediated immune response to tumor $c$

regulation of chromatin assembly or disassembly

regulation of cyclic nucleotide biosynthetic process

activated $\mathrm{T}$ cell apoptosis

regulation of Ras GTPase activity

chondroitin sulfate biosynthetic process

regulation of interleukin-3 biosynthetic process

phosphatidylcholine metabolic process

regulation of interleukin-1 beta secretion

positive regulation of alpha-beta $T$ cell activation

negative regulation of striated muscle development

positive regulation of nucleocytoplasmic transport 
regulation of neuron differentiation

positive regulation of gamma-delta T cell activation $\quad 0.25$

$\begin{array}{ll}\text { covalent chromatin modification } & 1.23\end{array}$

positive regulation of MHC class II biosynthetic process $\quad 0.25$

$\begin{array}{ll}\text { apoptotic program } & 1.97\end{array}$

negative regulation of cytokine biosynthetic process $\quad 0.49$

protein targeting to vacuole $\quad 0.25$

$\begin{array}{ll}\text { regulation of natural killer cell mediated immunity } & 0.25\end{array}$

prostanoid metabolic process $\quad 0.49$

$\begin{array}{ll}\text { insulin processing } & 0.25 \\ & 0.25\end{array}$

embryonic eye morphogenesis $\quad 0.25$

$\begin{array}{lr}\text { fucose catabolic process } & 0.49\end{array}$

regulation of epidermal growth factor receptor activity 0.49

neuron apoptosis $\quad 0.49$

negative regulation of gonadotropin secretion $\quad 0.25$

glycerophospholipid biosynthetic process $\quad 0.49$

\begin{tabular}{ll}
$T$ cell costimulation & 0.25 \\
\hline & 0.25
\end{tabular}

regulation of synaptic transmission, glutamatergic $\quad 0.25$

$\begin{array}{ll}\text { determination of anterior/posterior axis, embryo } & 0.25\end{array}$

positive regulation of interleukin-1 secretion $\quad 0.49$

neuroblast proliferation (sensu Vertebrata) $\quad 0.25$

GDP-mannose metabolic process

$\begin{array}{ll}\text { Docyte maturation } & 0.49\end{array}$

\begin{tabular}{ll} 
protein palmitoylation & 0.25 \\
\hline & 0.25
\end{tabular}

regulation of interleukin-8 biosynthetic process $\quad 0.25$

\begin{tabular}{ll} 
protein targeting to membrane & 0.25 \\
\hline
\end{tabular}

triacylglycerol mobilization $\quad 0.25$

$\begin{array}{ll}\text { positive regulation of Ras protein signal transduction } & 0.25\end{array}$

serotonin secretion $\quad 0.25$

spindle assembly $\quad 0.25$

arginine biosynthetic process $\quad 0.25$

chromatin disassembly $\quad 0.25$

endosome to lysosome transport $\quad 0.25$

regulation of chemokine biosynthetic process 0.25

positive regulation of gonadotropin secretion $\quad 0.25$

iron ion homeostasis $\quad 0.49$ 
negative regulation of cyclic nucleotide metabolic proce

inflammatory cell apoptosis

gluconeogenesis

embryonic gut morphogenesis

catecholamine secretion

sequestering of calcium ion

somatic recombination of immunoglobulin gene segmes

purine ribonucleoside triphosphate biosynthetic process

positive regulation of DNA replication

regulation of neurotransmitter secretion

tyrosine catabolic process

negative regulation of I-kappaB kinase/NF-kappaB cası

cysteine biosynthetic process

regulation of cellular $\mathrm{pH}$

regulation of follicle-stimulating hormone secretion

negative regulation of gliogenesis

modification by symbiont of host cell membrane

acetylcholine metabolic process

mRNA polyadenylation

succinyl-CoA pathway

immunoglobulin mediated immune response

cosanoid biosynthetic process

positive regulation of calcium-mediated signaling

negative regulation of MHC class II biosynthetic proces

ovarian follicle development

regulation of JNK cascade

CTP metabolic process

negative regulation of $B$ cell proliferation

protein targeting to mitochondrion

transmembrane receptor protein tyrosine kinase activat

NAD metabolic process

negative regulation of $\mathrm{T}$ cell proliferation

mitotic sister chromatid segregation

fibrinolysis

hemocyte differentiation (sensu Arthropoda)

negative regulation of DNA replication

\begin{tabular}{rl}
0 & 1 \\
0 & 1 \\
0 & 1 \\
0.67 & 1 \\
0 & 1 \\
0 & 1 \\
0 & 1 \\
1.33 & 1 \\
0 & 1 \\
0.67 & 1 \\
0 & 1 \\
0 & 1 \\
0 & 1 \\
0 & 1 \\
0 & 1 \\
0 & 1 \\
0 & 1 \\
0 & 1 \\
0 & 1 \\
0 & 1 \\
0.67 & 1 \\
0.67 & 1 \\
0 & 1 \\
0 & 1 \\
0 & 1 \\
0 & 1 \\
0.67 & 1 \\
0 & 1 \\
0 & 1 \\
0 & 1 \\
0 & 1 \\
0 & 1 \\
0.67 & 1 \\
0 & 1 \\
0 & 1 \\
0 & 1 \\
\hline & 1 \\
0 & 1 \\
0 & 1 \\
0 & 1 \\
0 & 1 \\
0 & 1 \\
0 & 1 \\
0 & 1 \\
0 & 1 \\
0 & 1 \\
0 & 1 \\
0 & 1 \\
0 & 1
\end{tabular}


regulation of odontogenesis (sensu Vertebrata)

positive regulation of phosphoinositide 3-kinase activity

positive regulation of striated muscle development

negative regulation of phosphorylation

negative regulation of MAPK activity

positive regulation of transcription factor activity

glycosphingolipid biosynthetic process

regulation of cAMP metabolic process

regulation of fatty acid oxidation

peptidyl-threonine phosphorylation

iron ion transport

copper ion transport

myeloid cell differentiation

regulation of interleukin-12 biosynthetic process

eye morphogenesis (sensu Vertebrata)

embryonic arm morphogenesis

Golgi to endosome transport

positive regulation of leukocyte degranulation

negative regulation of nucleotide biosynthetic process

male meiosis

peptidyl-diphthamide metabolic process

ATP metabolic process

RNA splicing, via transesterification reactions with bulgi

regulation of insulin receptor signaling pathway

establishment of T cell polarity

establishment of epithelial cell polarity

regulation of alpha-beta $T$ cell proliferation

cilium biogenesis

regulation of tumor necrosis factor-beta biosynthetic prc

complement activation

transformed cell apoptosis

regulation of interleukin-1 biosynthetic process

mitotic metaphase/anaphase transition

mitotic metaphase

histidine catabolic process 
pyrimidine ribonucleoside triphosphate biosynthetic pro $\quad 0.49$

regulation of interleukin- 4 biosynthetic process

female meiosis 0.49

$\begin{array}{ll}\text { positive regulation of fatty acid metabolic process } & 0.25\end{array}$

osteoblast differentiation 0.74

$\begin{array}{ll}\text { purine ribonucleoside diphosphate biosynthetic proces؟ } & 0.25\end{array}$

serotonin metabolic process $\quad 0.25$

$\begin{array}{ll}\text { eye photoreceptor cell differentiation } & 0.49\end{array}$

regulation of fibroblast growth factor receptor signaling

negative regulation of Ras protein signal transduction $\quad 0.25$

$\begin{array}{rll}0 & 1 & 1 \\ 0 & 1 & 1 \\ 0 & 1 & 1 \\ 0.67 & 1 & 1 \\ 0.67 & 1 & 1 \\ 0 & 1 & 1 \\ 0 & 1 & 1 \\ 0 & 1 & 1 \\ 0.67 & 1 & 1 \\ 0 & 1 & 1 \\ 0 & 1 & 1 \\ 0.67 & 1 & 1 \\ 0 & 1 & 1 \\ 0 & 1 & 1\end{array}$

